\title{
Biradical Formation by Deprotonation in Thiazole-Derivatives: The Hidden Nature of Dasatinib
}

Carlos Heras, Daniel Reta, Rosendo Valero, Guillermo Albareda, Nicholas Chilton, Alistair Fielding, Iberio de P. R Moreira, Josep Maria Bofill, Francisco Lopez Calahorra

Submitted date: 31/10/2019 - Posted date: 06/11/2019

Licence: CC BY-NC-ND 4.0

Citation information: Heras, Carlos; Reta, Daniel; Valero, Rosendo; Albareda, Guillermo; Chilton, Nicholas; Fielding, Alistair; et al. (2019): Biradical Formation by Deprotonation in Thiazole-Derivatives: The Hidden Nature of Dasatinib. ChemRxiv. Preprint.

The formation of stable organic biradicals by a deprotonation process is reported for a series of conjugated heterocycles that share a Ph- $\mathrm{N}(\mathrm{H})$-2-thiazole structural motif. We characterise the paramagnetic electronic ground state by means of continuous-wave and pulse EPR. We propose a simple valence bond mechanism for a deprotonation-induced formation of paramagnetic organic molecules, based on the interplay between the electronegativity of heteroatomic groups and the recovery of aromaticity to stabilise the biradical species. The $\mathrm{Ph}-\mathrm{N}(\mathrm{H})$-2-thiazole motif is found in a variety of biologically active molecules, exemplified here with the anticancer drug Dasatinib, and our results suggest a radical-based mechanism for the protein kinase inhibition activity of the drug. The existence of this structure-property relationship for an elementary chemical motif suggests that biradical species may be more prevalent than previously thought and have an important role in bioorganic chemistry.

File list (2)

Biradicals_v8.docx (1.25 MiB) view on ChemRxiv - download file Biradicals_v8_ESI.docx (4.78 MiB) view on ChemRxiv - download file 


\title{
Biradical formation by deprotonation in thiazole-derivatives: the hidden nature of Dasatinib
}

\author{
Carlos Heras, ${ }^{1}$ Daniel Reta, ${ }^{2 *}$ Rosendo Valero ${ }^{3,4}$ Guillem Albareda, ${ }^{4,5}$ Nicholas \\ F. Chilton, ${ }^{2}$ Alistair J. Fielding, ${ }^{5}$ Ibério de P. R. Moreira, ${ }^{3,4^{*}}$ \\ Josep Maria Bofill, ${ }^{1,4}$ and Francisco López-Calahorra, ${ }^{1}$ \\ ${ }^{1}$ Departament de Química Inorgànica i Orgànica, Sec. de Química Orgànica, Universitat de \\ Barcelona, C/ Martí i Franquès 1, E-08028 Barcelona, Spain. \\ ${ }^{2}$ School of Chemistry, The University of Manchester, \\ Oxford Road, Manchester, M13 9PL, United Kingdom. \\ ${ }^{3}$ Departament de Ciència dels Materials i Química Física, Sec. de Química Física, Universitat \\ de Barcelona, C/ Martí i Franquès 1, E-08028 Barcelona, Spain. \\ ${ }^{4}$ Institut de Química Teòrica i Computacional (IQTCUB), \\ Universitat de Barcelona, C/ Martí i Franquès 1, E-08028 Barcelona, Spain. \\ ${ }^{5}$ Max Planck Institute for the Structure and Dynamics of Matter and Center for Free-Electron \\ Laser Science, Luruper Chaussee 149, 22761 Hamburg, Germany \\ ${ }^{6}$ School of Pharmacy and Biomolecular Sciences, Liverpool John Moores University, Byrom \\ Street, Liverpool, L3 3AF, United Kingdom.
}

\begin{abstract}
The formation of stable organic biradicals by a deprotonation process is reported for a series of conjugated heterocycles that share a $\mathrm{Ph}-\mathrm{N}(\mathrm{H})$-2-thiazole structural motif. We characterise the paramagnetic electronic ground state by means of continuous-wave and pulse EPR. We propose a simple valence bond mechanism for a deprotonation-induced formation of paramagnetic organic molecules, based on the interplay between the electronegativity of heteroatomic groups and the recovery of aromaticity to stabilise the biradical species. The $\mathrm{Ph}-\mathrm{N}(\mathrm{H})$-2-thiazole motif is found in a variety of biologically active molecules, exemplified here with the anticancer drug Dasatinib, and our results suggest a radical-based mechanism for the protein kinase inhibition activity of the drug. The existence of this structure-property relationship for an elementary chemical motif suggests that biradical species may be more prevalent than previously thought and have an important role in bioorganic chemistry.
\end{abstract}

\section{Introduction}

Most organic molecules present an even number of electrons distributed over doubly occupied molecular orbitals, resulting in diamagnetic ground states. However, applications derived from introducing unpaired electrons and thus magnetism into organic molecules are relevant across various fields, such as redox-biology, ${ }^{1}$ optical devices, ${ }^{2}$ reaction mechanisms, ${ }^{3}$ multifunctional materials, ${ }^{4}$ or spintronics. ${ }^{5}$ Following the discovery of Gomberg's triphenylmethyl radical ${ }^{6}$ and of Thiele $^{7}$ and Tschitschibabin $^{8}$ diradicals, a route to preventing electron-pairing and stabilising paramagnetic electronic states in organic molecules was developed. These works showed that careful design of the molecular topology ${ }^{9}$ can lead to an electronic structure with two unpaired electrons weakly interacting in two nearly-degenerate nonbonding molecular orbitals ${ }^{10,11}$ - the relative stabilization of the resulting spin-triplet 
(diradical) vs the spin-singlet (quinoidal) states is dictated by an aromaticity gain on the cyclic subunits that links the radical centres (Scheme 1a). ${ }^{12,13}$

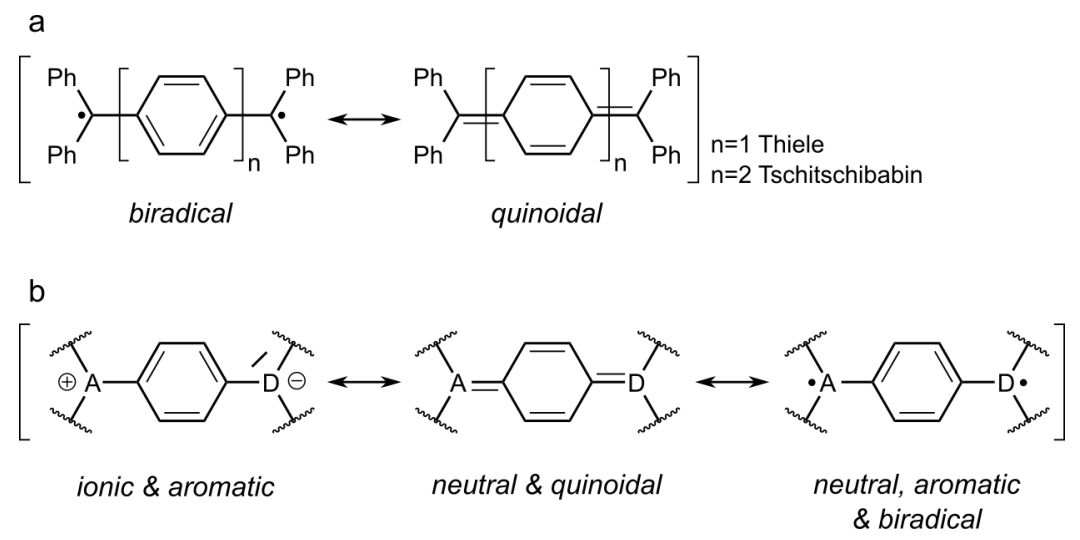

Scheme 1: Dominant valence bond forms in the electronic ground state of a) Thiele and Tschitschibabin hydrocarbons and b) heteroatom-containing (A, D) Thiele anion analogue. Unpaired electrons are indicated with dots.

EPR measurements show that in Tschitschibabin's ${ }^{14-15161718}$ diradical, the energy separation between the ground spin-singlet and the excited spin-triplet states is thermally accessible ( $\Delta E_{S-T} 2.5 \mathrm{Kcal} / \mathrm{mol}$ ) and MO calculations suggest the gap is $5.5 \mathrm{Kcalmol}^{-1}$ in the Thiele hydrocarbon. ${ }^{19,20}$. Following these early works, this approach for designing stable organic radicals has been successfully applied to the general class of even alternant polyaromatic hydrocarbons (PAHs) ${ }^{21}$ achieving almost degenerate spin states ( $\Delta E_{S-T} 0$ ), and more recently to analogues with unpaired electrons hosted on carbene- ${ }^{22}$ and nitrogen- ${ }^{23}$ centers, where $\Delta E_{S-T}$ can be modulated by varying the substituents - all these systems are symmetric, and to the best of our knowledge this approach has not been tested in asymmetric molecules. Importantly, these studies demonstrate that in principle, an appropriate linkage of adequate $\pi$-conjugated systems has the potential to yield organic molecules with diradical ground states. However, these systems contain only the most basic aspects that affect the molecular electronic structure, obviating multiple other features such as structural flexibility or the presence of multiple heteroatoms. Notably, heteroatoms can introduce ionic forms to accommodate unpaired electrons in otherwise inaccessible resonant paths, therefore offering promising, yet widely unexplored mechanisms to stabilize biradical states (Scheme 1b). In this simplified topological description, the two different heteroatoms (or heteroatomic groups, A and D) with different electronegativities, are connected via an aromatic bridging (ortho- or para-) ring that allows for their resonant interaction. The interplay between the groups A and D with the molecular charge offers resonant paths that are topologically equivalent (with a different charge distribution) to those obtained in Thiele's diradical, allowing for a redistribution of the electronic charge density on the molecule. In a first step, an anion is formed leading to a large electron density accumulated in $\mathrm{D}$, which is alleviated by a charge transfer to the more electropositive A moiety, resulting in a neutral quinoidal form. Finally, the recovery of aromaticity drives the process toward the neutral 
aromatic diradical, which can be further stabilised if the generated unpaired electrons can delocalise over the corresponding heteroatomic group. Thus, one might wonder whether more generic classes of molecules showing these extra degrees of freedom can also stabilise open-shell ground states.

In this work we set out to investigate this question and propose an alternative general approach to maximise the contributions that stabilise biradical ground states in organic molecules. In particular, we investigate a series of thiazole-based derivatives (Figure 1), as they are a general molecular class fulfilling the discussed topological arguments, have been investigated by some of us over the last years ${ }^{24,25}$ and most importantly, present interesting reactivity profiles being used as anticancer ${ }^{26-27282930}$ and antiviral $^{31,32}$ therapeutic agents. The herein proposed model has implications to understand the processes involved in the reactivity of these biologically relevant molecules, as exemplified by the results on $\mathbf{4}$, the anticancer drug commercially known as Dasatinib (®Sprycel).

\section{Results and discussion.}

Compounds 1-4 (Figure 1) offer a set of molecules where the effects that contribute to the proposed biradical formation can be addressed separately, as they are introduced to varying degrees in each molecule. The synthesis and characterisation of compounds 1-3 is described in section 1 of the Supplementary Information (SI) - 4 was purchased, kept refrigerated and measured without any further manipulation. Common to all 1-4 is a structural consisting of $i$ ) an electron deficient aromatic group $\left(\mathrm{NO}_{2}\right.$ in 1-3, amido group in 4), ii) an electron donating aromatic group (thiazol group), and iii) a tautomeric enamine-imine linking group that results in amphoteric properties (1,4-phenylene in 1, 2,6-acridine in 2 and 3, and 2,4-thiazolyl in 4 ). The amphoteric character of molecules $\mathbf{1}, \mathbf{2}$ and $\mathbf{4}$ arises from the tautomerism of the imino-amino bridging group (also the amido group in $\mathbf{4}$ ), whereas in molecule $\mathbf{3}$ it results from the acidity of the $\mathrm{H}$ atoms of the methyl group attached to the amine, that is largely increased by the adjacent positively charged nitrogen due to inductive and resonant effects. These are well-known effects ${ }^{33}$ (Section SI 4 for details). In this case, this acidic proton can be exchanged between the methyl group and the imino-amino group bridging the thiazole and acridine moieties and is responsible of its amphoteric character. 
<smiles>Cc1nc(Nc2ncc(C(=O)Nc3c(C)cccc3Cl)s2)cc(N2CCN(CCO)CC2)n1</smiles>

Figure 1: Schematic representation of 1-4. Green, red and black bonds indicate electron deficient, electron donating and tautomeric linking groups (Scheme 1), respectively. 4 is known as Dasatinib.

To test whether the structural and electronic elements in 1-4 are effective at inducing biradical formation, we probe the electronic ground states of 1-4 via continuous-wave electron paramagnetic resonance (cw-EPR) spectroscopy, which selectively investigates the transitions associated with unpaired electrons. Variable temperature X-band ( 9.4 GHz) EPR spectra were recorded for powder samples of all compounds (Figure 2, top row), showing a sharp isotropic transition centred at $~ 330$ $\mathrm{mT}$ ( $\mathrm{g} \sim 2$ ), characteristic of organic radicals. The most intense signal (overall these are weak signals) is consistently obtained at the lowest temperature, and the signal reduces exponentially with increasing temperature; this is a typical feature arising from the Boltzmann population in a paramagnetic ground state. Molecules $\mathbf{2}$ and $\mathbf{3}$ show the most intense signals over the whole temperature range (based on signal-tonoise ratio), which we assign to a more effective delocalisation of the unpaired electrons over the extended conjugated $\pi$-system of acridine. Molecules $\mathbf{1}$ and $\mathbf{4}$ also show clear EPR transitions, but these are less intense due to the weaker electron withdrawing groups and the limited delocalisation over smaller $\pi$-systems. An EPR signal remains for $\mathbf{4}$ up to room temperature (Figure S5), but is omitted from Figure 2 for temperatures above $30 \mathrm{~K}$ for clarity. 

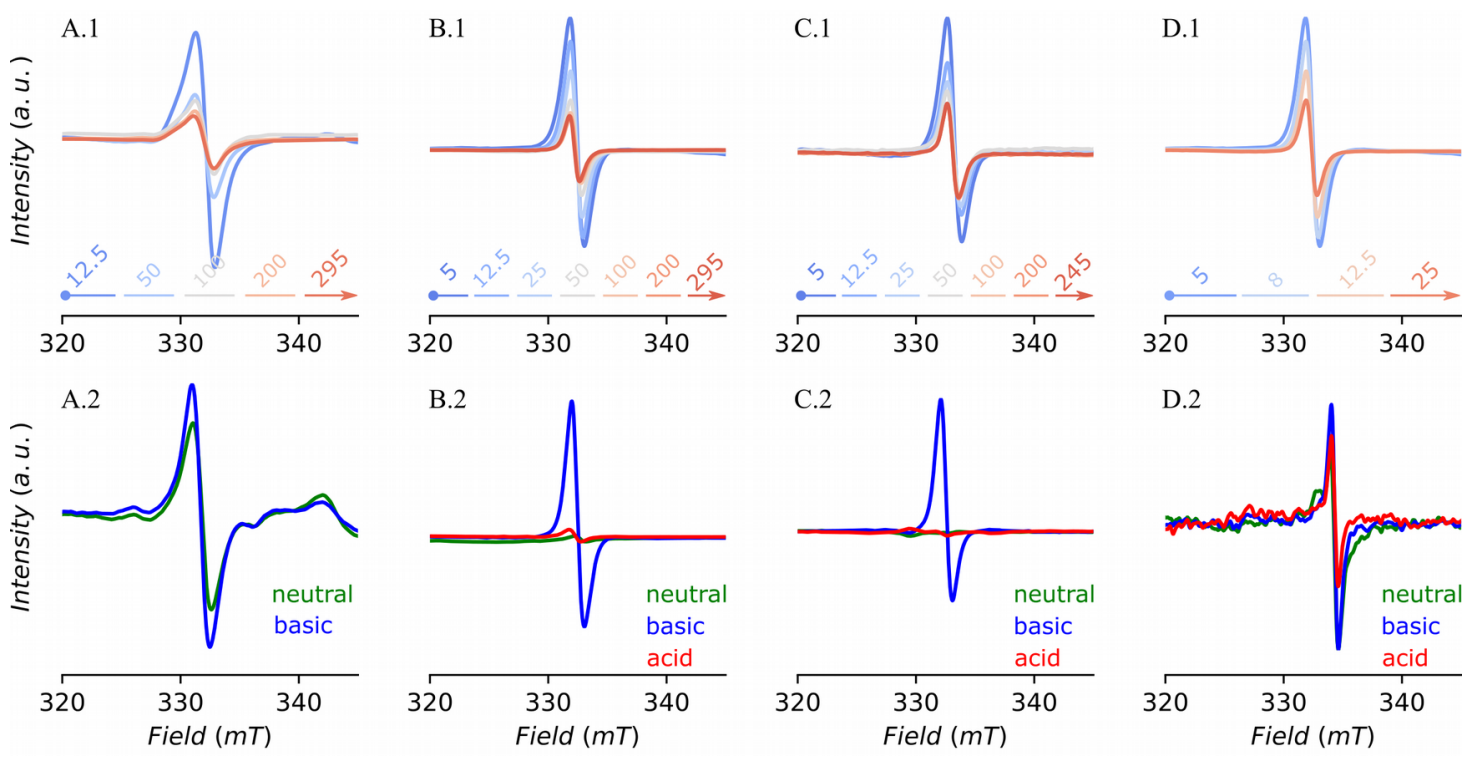

Figure 2: Normalised X-band EPR spectra for compounds 1-4, A-D, respectively. Top row reports the temperature dependent powder spectra. Bottom row presents frozen solution spectra (12.5 K for 1, $5 \mathrm{~K}$ for 2-4) of the neutral, deprotonated and acidified samples.

Frozen solution X-band EPR measurements (Figure 2, bottom row and Figure S3) were performed to $i$ ) to probe the intramolecular origin of the observed signals in the powder measurements, and ii) to investigate the effect of deprotonation on the biradical character. Powder samples of $\mathbf{1}$ and 2-4 were dissolved in the aprotic solvents dichloromethane (DCM) and dimethylsulfoxide (DMSO), respectively, and degassed under a positive flow of $\mathrm{He}$; all tubes were sealed with rubber caps to prevent contamination with paramagnetic molecular oxygen. An aprotic solvent is needed to avoid interference with the subsequent addition of a base to induce deprotonation. Frozen solution EPR spectra (labelled neutral in Figure 2) show signals at the same field position with comparable linewidths to the ones obtained in powder, confirming the intramolecular origin of the paramagnetic species. What appears to be fine structure in the frozen solution spectrum of $\mathbf{1}$ is the result of a weak signal after background-subtraction (see background in Fig S4). Molecule 3 also shows a very weak signal due to the small amount of sample that could be dissolved.

To probe the effect of deprotonation, approximately $50 \quad \mu \quad \mathrm{L}$ of the strong base 1,8diazabicyclo[5.4.0]undec-7-ene (DBU) was added to $\sim 150 \quad \mu$ L of neutral solution with a syringe through the rubber caps directly into the X-band tubes, under a positive flow of He. After mixing, the tubes were put back into the exact same position of the resonator. For all four samples, the colour of the solution drastically darkened on addition of base (Figure S24) and we observed a consistent increase in the EPR signal intensity (labelled basic in Figure 2) measured at the same temperature and position in the resonator.

Subsequent addition of acetic acid (labelled acid in Figure 2) reverts the process and results in either a reduction of the signal with respect to the basic solution (4), or its 
almost entire suppression (2-3). For 1, acid was not added because the last batch of that sample was left open to air and the signal decayed. In addition to the reduction of the EPR signal, the solution reverted to its original colour upon addition of acid (Figure S24).

To gain further insight on the effect of deprotonation and to connect the radical signals unambiguously to the structural motif in these molecules, we synthesised an analogue that shares the structural elements of 1-4 but lacks the acidic proton due to the absence of imine-enamine tautomerism. Figure 3 shows the X-band EPR silent spectra at $5 \mathrm{~K}$ of the powder and frozen DMSO solutions (6.55 mg dissolved in 200 $\mu \mathrm{L}$ of anhydrous DMSO), confirming that only the anionic form fulfils the topological conditions to host unpaired electrons, i.e., an acidic proton is crucial to stabilise a biradical configuration (see Figure S7 for more details). Note that the intensity scale is orders of magnitude smaller than for $\mathbf{1 - 4}$, using a similar amount of sample.

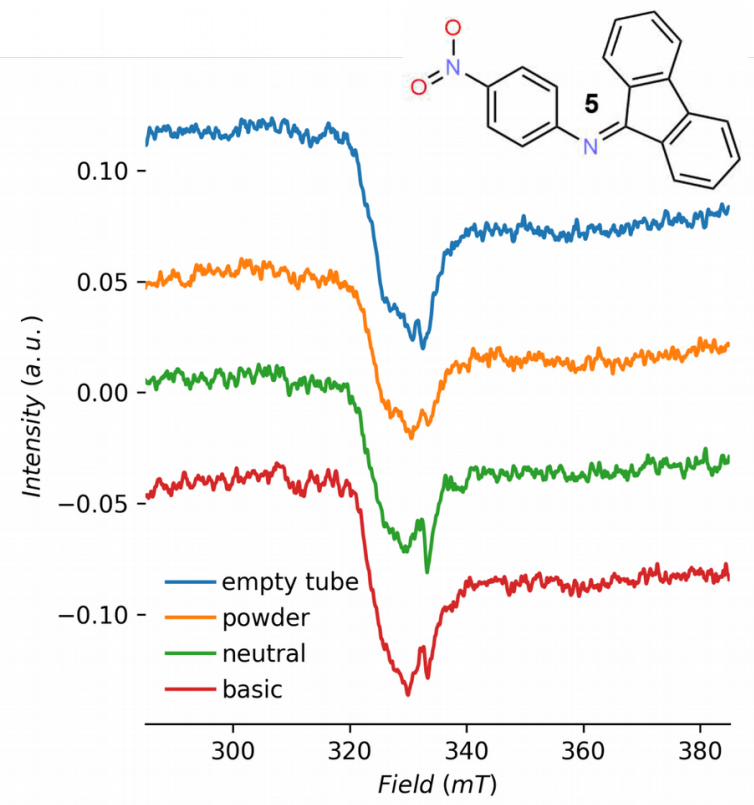

Figure 3: X-band EPR spectra of powder and frozen solutions of compound 5 at $5 \mathrm{~K}$.

Having clarified that the anion is responsible for the paramagnetic response, it remains to determine if it is a mono- or biradical. The discrimination of these two situations is crucial since each one implies fundamentally different processes and mechanisms of formation, and has different properties. Unfortunately, the isotropic character of all EPR signals together with the absence of "half-field" or "forbidden" transitions (from the $m_{S}=-1$ to +1 of a possible biradical $S=1$ ) can be explained assuming either a monoradical or a biradical with a very weak interaction between the two electrons, as indicated by our simulations (Section SI 2.1). The latter case has been observed before in bisgalvinoxyl, ${ }^{34}$ where the EPR spectrum is characteristic of two independent doublets, and more recently in trioxotriangulene dimers, ${ }^{35}$ where halffield transitions are not observed despite a confirmed triplet ground state. Thus here, we do not have the experimental evidence to say one way or the other, but we can 
rationalise that these are biradicals with established chemical and reactivity arguments. We reason that if a mono-radical is created when the base is added, it must happen through a homolytic rupture of a labile bond in the molecule, leading to radical fragments that would rapidly react with other molecules - those could be identified in the final solution after neutralisation (e.g. by NMR), and would be different from the original solution. If instead a biradical is generated, the process could be reverted because no chemical bond needs to be broken. Figures S22-27 present the UV-Vis and NMR spectra of the neutral, basic and acidic solutions, showing that the pure and neutralised solutions are spectroscopically identical, whereas the intermediate basic solution shows distinctly different features. Finally, the possibility of an intermolecular biradical by an electron transfer in a pair of molecules is also not plausible, given the consistency of the results across molecules and solvents with different permittivity (1 in DCM, 2-4 in DMSO). This, together with the increase in the EPR signal upon addition of base, confirms that the generation of the radicals is reversible and discards the possibility of a monoradical. Thus, a deprotonation-induced molecular biradical is the most consistent explanation of the observed paramagnetic response.

The measured data can be qualitatively explained using the topological and valence bond description presented in Scheme 2a. We discuss our model for $\mathbf{1}$, but we theorise that it is a general mechanism applicable to any molecule containing the structural motif of Scheme 1b. Similar valence bond analysis for compounds $\mathbf{2 - 4}$ are in section 3 of the SI. Analogously to diamines or imidazole, the imine-enamine tautomerism of to the bridging nitrogen and the thiazole unit makes these molecules amphoteric, providing a mechanism for a fraction of the molecules to generate an anion from a proton transfer between two neutral molecules. In the solid state (powder measurements), the biradical species can be generated by a proton transfer between two identical amphoteric molecules during the formation of the solid as $\begin{gathered}+i \\ -i+A H_{2}^{i} \\ 2 A H \leftrightarrow A^{i}\end{gathered}$ by precipitation from the solution. Thus, different precipitation methods mean different amounts of paramagnetic $A^{i}$ being formed, as indicated by the magnetisation saturation values going from 84 to $1.2 \mathrm{emu} \cdot \mathrm{mol}^{-1}$ for $\mathbf{1}$ (Figure S19, measured on the same sample batch), depending on the precipitation method employed. In solution, the formation of the anion by this equilibrium is less favourable but still provides a defined fraction of deprotonated form at a given temperature - that fraction is largely increased in a basic medium (i.e. by adding DBU and ensuring an aprotic solvent). Additionally, the structural flexibility of the bridge allows for an outof-plane conformation between the two aromatic groups, which could stabilise the biradical due to orthogonality of the magnetic orbitals. ${ }^{36}$ Scheme 2 a introduces the valence bond forms involved in the formation of the biradical $\mathbf{1}$ - among them, the one with the unpaired electrons on the bridging nitrogen and the $\mathrm{NO}_{2}$ moiety is dominant due to a greater aromatic recovery (highlighted in blue square). Molecule $\mathbf{3}$ does not 
present an imine-enamine tautomerism and the formation of the anion involves the acidic methyl hydrogens instead (section 4 of the SI). Nonetheless, the stabilisation of the biradical form is based on the same electronic processes as in the other molecules.

To obtain a better description of the biradical formation, we performed wave function and density functional theory calculations on $\mathbf{1}$, as it is the simplest molecule (see Section S4 for details). However, all our approaches fail at predicting a consistent triplet-singlet gap, which is highly dependent on geometries, active spaces and basis sets; this speaks of the complexity of the problem, resulting from a critical combination of strong electronic correlation and electron-nuclear coupling effects. We suggest that the reversible deprotonation process induces a region of highly correlated electron-nuclear motion, allowing for a complex crossing of quasi-degenerate electronic states with different spin multiplicity (see Scheme 2b). Therefore, any attempt to describe this process cannot rely on the Born-Oppenheimer approximation of electron-nucleus separability, rendering any standard computational method inapplicable. Adding to this, one cannot include static electronic correlation in a balanced way for the two spin states $\left(S_{0}\right.$ and $T_{0}$ ) with a reasonably sized active space due to the highly delocalized nature of the problem - as a result of this imbalance the singlet-spin state is artificially stabilized in our calculations. For instance, in similar compounds, ${ }^{37,3839}$ spin-polarisation arising from the $\sigma$-backbone needs to be treated as a first-order effect, making impractical any wavefunction-based approach to include further dynamic correlation or to construct a potential energy surface to perform a dynamics calculation. 
Scheme 2. a) Valence bond forms in the ground state of the anion of compound 1. Unpaired electrons are represented by dots and the dominant biradical form is marked in blue. Red lone pairs cannot delocalise due to $\sigma-\pi$ orthogonality. b) Model for the energy profile of the proton extraction process, responsible for the biradical formation. Darkened area indicates the region of strong electron-nucleus coupling and quasi-degenerate states with height of the order of $k_{B} T$.

In our proposed model, the unpaired electrons are mainly hosted by nitrogen and one would expect to observe hyperfine splitting due to the nuclear spin $I=1$ of ${ }^{14} \mathrm{~N}$, in apparent contradiction with the measured cw-EPR data. However, considering the experimental EPR linewidth and hyperfine constants in line with literature values one can simulate an EPR spectrum with no hyperfine features (Figure S9). To gain further evidence towards the presence of ${ }^{14} \mathrm{~N}$ hyperfine interaction and the validation of the model, pulse X-band experiments were performed for $\mathbf{2}$ and 3; the signal for $\mathbf{4}$ is too weak to detect and we did not have more sample of $\mathbf{1}$. Echo-detected field-swept (EDFS) spectra for $\mathbf{2}$ and $\mathbf{3}$ are presented in Figure S10-11, respectively. Compound $\mathbf{2}$ shows an intense transition at $\mathrm{H}_{\mathrm{dc}}=3550 \mathrm{G}$ and a broad peak at $\mathrm{H}_{\mathrm{dc}}=3454 \mathrm{G}$, the latter being obscured in the cw-EPR. Collection of an EDFS spectrum at the same conditions of the empty resonator and empty tube confirm that this is not an artefact of the resonator. The derivative of the EDFS spectrum is consistent with that obtained from cw-EPR (Figure S12). The intense and broad transitions are assigned to each of the electrons in the biradical, one mainly localised on the bridging nitrogen and the 
other delocalised over the acridine and nitro group (Figure S29). Conversely, compound 3 shows a single intense transition at $\mathrm{H}_{\mathrm{dc}}=3529 \mathrm{G}$, with no indication of the broad peak. In this case, the deprotonated methyl group participates of all resonant paths (Figure S30), resulting in a comparatively more delocalised unpaired electron which is not observed. For each maximum in the EDFS spectra of $\mathbf{2}$ and $\mathbf{3}$, we performed 2- and 3-pulse X-band ESEEM experiments (Figure S13-16) to access the nuclear quadrupole interactions and obtain estimates of the nuclear ${ }^{14} \mathrm{~N}$ hyperfine parameters; the advantage of a 3-pulse sequence is the absence of band combinations, which results in a cleaner spectrum. Figure 4 presents the frequency-domain 3-pulse ESEEM data for 3, where the three nuclear quadrupole resonances and the double quantum transitions are identified, in line with reported values. ${ }^{40-41424344}$ This provided a good starting point to fit the 3-pulse and simulate the 2-pulse data (Figure S17, see section 2.1.1 for details). In conclusion, we consistently observe the fingerprints of ${ }^{14} \mathrm{~N}$ in $\mathbf{2}$ and 3, which provides strong evidence towards the validity of the proposed model for the generation of the molecular biradical - this can be safely extrapolated to $\mathbf{1}$ and 4 due to the consistency of the different spectroscopic results.

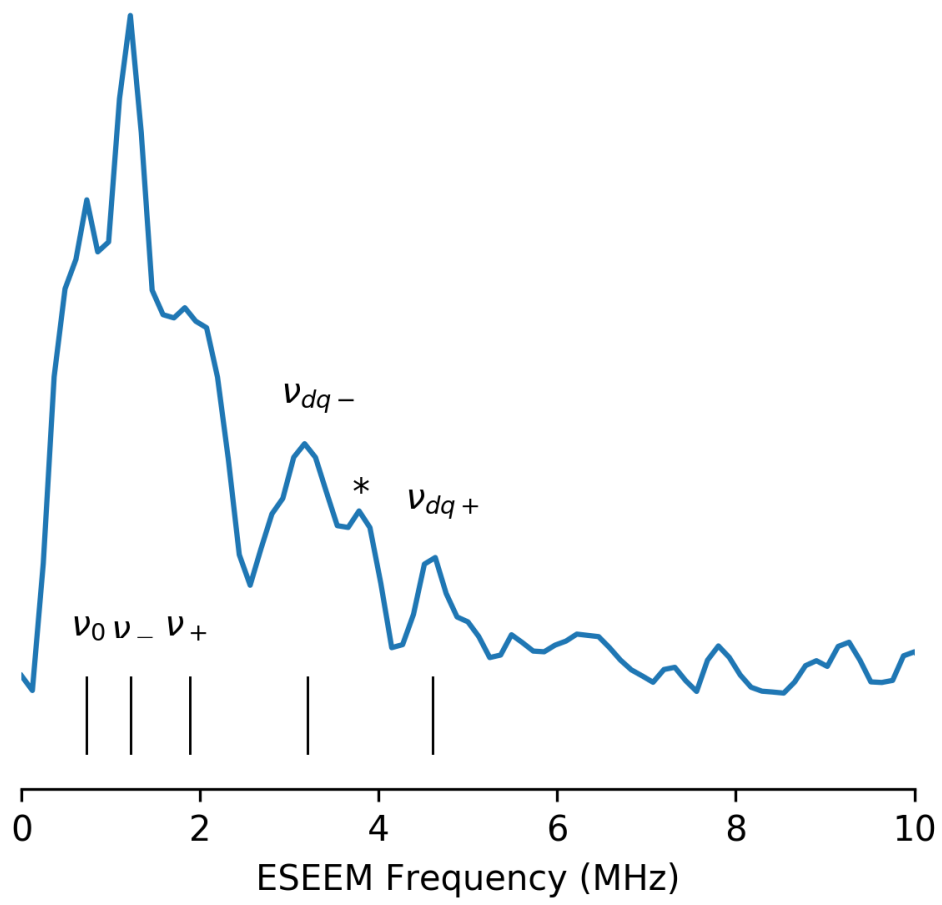

Figure 4. X-band frequency-domain of 3-pulse electron spin echo envelope modulation (ESEEM) for 3 at $5 \mathrm{~K}$ and $352.9 \mathrm{mT}$ field position. * indicates the Larmor frequency ${ }^{13} \mathrm{C}$.

The observed radical character of compound 4 (Dasatinib), deserves special attention. Dasatinib is an anticancer drug that binds to tyrosine kinase proteins and prevents cancer cells from multiplying. ${ }^{45}$ It is used for treatment of Imatinib-resistant chronic myelogenous leukemia and is now being evaluated for use in advanced prostate and breast cancer. ${ }^{46,47}$ Its mechanism of action is explained in terms of competitive interactions with the catalytic pocket or through allosteric interactions 
with other regions of the kinase, ${ }^{46}$ i.e. through electrostatic and dispersion forces. However, its anomalously high selectivity and efficiency could be better explained considering the formation of a local covalent bond via a radical-based mechanism. In fact, single crystal X-ray diffraction of the drug bound to the protein kinase ${ }^{48}$ reveal that the bond distances of the C-N-C Dasatinib bridge are $1.37 \AA$, in between the distance expected for imine (C=N, $1.25 \AA$ ) and amine (N-C, $1.43 \AA$ ) bonds, ${ }^{49}$ matching a conjugated $C \cdots N \cdots C$ bond as predicted by our model. Additionally, recent unexpected results on "exceptional cytotoxicities" of imido-ferrociphenols against triple-negative breast cancer (TNBC) cells have been correlated with the $\pi$ system of their quinone methides constituents, ${ }^{50}$ which interestingly share the same topological ingredients here discussed. Although speculative on its own, these observations become relevant in light of our EPR data. 


\section{Conclusions}

We have characterised the molecular biradical nature of a series of thiazolederivatives and assign the effect to a specific structural pattern. The biradical character is manifested in the anionic state of the molecules, further confirmed by EPR silent experiments on an analogue that does not possess an acidic proton. The unpaired electrons show interaction with the nuclear spin of ${ }^{14} \mathrm{~N}$, as determined by pulsed EPR on 2 and 3. UV-Vis and NMR spectroscopy is employed to demonstrate that the protonation-deprotonation process is reversible, resulting in a stable biradical. We rationalise our findings based on a valence bond description of the available resonant forms in which the interplay between aromaticity of the bridging ring and molecular charge is key, and hypothesise that any molecule presenting the discussed structural motif should present a biradical character. The importance of this finding is exemplified by the results on Dasatinib (4), suggesting overlooked radical-based mechanisms for its biological activity. Altogether, our results call for a change of paradigm in the chemistry of thiazole-derived compounds. 


\section{Acknowledgments}

This research was supported by the Spanish MINECO, the Agencia Estatal de Investigación (AEI), Fondo Europeo de Desarrollo Regional (FEDER; CTQ201676423-P), Spanish Structures of Excellence María de Maeztu program (MDM-20170767, Generalitat de Catalunya (grant 2017SGR348 and XRQTC), and the UK EPSRC (EP/P002560/1). G.A. acknowledges also financial support from the European Union's Horizon 2020 research and innovation programme under the Marie Skłodowska-Curie grant agreement No 752822. We thank the EPSRC National EPR Facility at The University of Manchester for EPR measurements, along with Prof. Eric McInnes, Prof. David Collison, Dr. Marie-Emmanuelle Boulon, Mr. Edmund Little and Mr. Adam Brookfield. We thank Alexis San Juan for NMR spectra. 
References 
1 K. V. Avondt, E. Nur, S. Zeerleder, Nature Reviews Nephrology, 2019, 15, 671 - 692.

2 X. Ai, E. W. Evans, S. Dong, A. J. Gillett, H. Guo, Y. Chen, T. J. H. Hele, R. H. Friend, F. Li, Nature, 2018, 563, 536-540.

3 D. Leifert, A. Studer, Angew. Chem., Int. Ed. 2019, doi.org/10.1002/anie.201903726.

4 J. Veciana, I. Ratera, Chapter 2 in 2010, John Wiley \& Sons, Ltd, ISBN:9780470666975.

5 S. Sanvito, Chem. Soc. Rev., 2011, 40, 3336-3355.

6 M. Gomberg, J. Am. Chem. Soc. 1900, 22 (11), 757-771.

7 J. Thiele, H. Balhorn, Ber. Dtsch. Chem. Ges. 1904, 37, 1463-1470.

8 A. E. Tschitschibabin, Ber. Dtsch. Chem. Ges. 1907, 40, 1810-1819.

9 I. Gutman, O. E. Polansky, Springer-Verlag: New York, 1986.

10 L. Salem, C. Rowland, Angew. Chem., Int. Ed. Engl. 1972, 11, 92-111.

11 M. Abe, Chem. Rev. 2013, 113, 7011-7088.

12L. K. Montgomery, J. C. Huffman, E. A. Jurczak, M. P. Grendze, J. Am. Chem. Soc. 1986, 108, 6004-6011.

13 G. Trinquier, J. P. Malrieu, Chem. Eur. J., 2015, 21, 2, 814-828.

14 C. A. Hutchison, A. Kowalsky, R. C. Pastor, G. W. Wheland, J. Chem. Phys. 1952, 20, 1485.

15 D. C. Reitz, S. I. Weissman, J. Chem. Phys. 1960, 33, 700.

16 R. K. Waring, G. J. Sloan, J. Chem. Phys. 1964, 40, 772.

17 H. M. McConnell, J. Chem. Phys. 1960, 33, 1868.

18 E. Muller, A. Rieker, K. Scheffler, A. Moosmayer, Angew. Chem. internat. Edit. 1966, 5, 1.

19 H. S. Jarrett, G. J. Sloan, W. R. Vaughan, J. Chem. Phys. 1956, 25, 697.

20 I. D. Morozova, M. E. Dyatkina, Russ. Chem. Rev. 1968, 37, 376.

21 Z. Zeng, X. Shi, C. Chi, J. T. Lopez Navarrete, J. Casado, J. Wu, Chem. Soc. Rev., 2015, 44, 6578-6596.

22 D. Rottschäfer, N. K. T. Ho, B. Neumann, H. G. Stammler, M. van Gastel, D. M. Andrada, R. J. Ghadwal, Angew. Chem. Int. Ed. 2018, 57, 5838 -5842

23 G. Tan, X. Wang, Acc. Chem. Res. 2018, 50, 1997-2006.

24A. Molinos-Gómez, X. Vidal, M. Maymó, D. Velasco, J. Martorell, F. López-Calahorra, Tetrahedron 2005, 61, 9075 -9081.

25S. Latorre, I. de P. R. Moreira, B. Villacampa, L. Julià, D. Velasco, J. M. Bofill and F. López-Calahorra, ChemPhysChem 2010, 11, 912-919.

26 T. C. Mahesh, S. Patel, P. Modi, P. S. Brahmkshatriya, Current Topics in Medicinal Chemistry, 2016, 16, 26, $2841-2862$.

27 M. D. Altıntop, B. Sever, B. A. Çiftçi, A. Özdemir, Molecules. 2018, 23(6): 1318.

28 A. Jamwal, A. Javed, V. Bhardwaj, J. Pharm. BioSci. 2013, 3, 114-123.

29 S. Jain, S. Pattnaik, K. Pathak, S. Kumar, D. Pathak, S. Jain, A. Vaidya, Mini-Reviews in Medicinal Chemistry 2018, 18, 8, 640 - 655.

30 T. Italo de Santana, M. Barbosa, P. A. T. de M. Gomes, A. C. N. da Cruz, T. Gonçalves da Silva, A. C. L. Leite, European Journal of Medicinal Chemistry 2018, 144, 874-886.

31 K. M. Dawood, T. M. A. Eldebss, H. S. A. El-Zahabi, M. H. Yousef, European Journal of Medicinal Chemistry 2015, 102, 266-276.

32 O. I. El-Sabbagh, M. M. Baraka, S. M. Ibrahim, C. Pannecouque, G. Andrei, R, Snoeck, J. Balzarini, A. A. Rashad, European Journal of Medicinal Chemistry, 2009, 44, 9, 3746-3753.

33 E. A. Anslyn, D. A. Dougherty, Modern Physical Organic Chemistry,University Science Books, Sausalito 2008 ISBN: 978-1-891389-31-3

34 E. A. Chandross, J. Am. Chem. Soc. 1964, 86, 1263.

35 T. Murata, N. Asakura, S. Ukai, A. Ueda, Y. Kanzaki, K. Sato, T. Takui, Y. Morita, ChemPlusChem 2019, 84, $1-7$.

36 L. Salem, John Wiley \& Sons, Inc. New York, 1982, pp 90-91.

37 N. Ferre, N. Guihery, J. P. Malrieu, Phys. Chem. Chem. Phys., 2015, 17, 14375-14382.

38 J. P. Malrieu, R. Caballol, C. Calzado, C. J. de Graaf, N. Guihery, Chem. Rev. 2014, 114, 429-492.

39 G. Trinquier, J. P. Malrieu, J. Phys. Chem. A 2018, 122, 6926-6933.

40 S. R. Rabbani, D. T. Edmonds, P. Gosling, M. H. Palmer, Journal of Magnetic Resonance 1987, 72, 2, 230-237.

41 D. T. Edmonds, P. A. Speight, Physics Letters A 1971, 34, 6, 325-326.

42 A. T. Taguchi, P. J. O’Malley, C. A. Wraight, S. A. Dikanov, J. Phys. Chem. B 2014, 118, 31, 9225-9237.

43 J. McCracken, S. Pember, S. J. Benkovic, J. J. Villafranca, R. J. Miller, J. Peisach, J. Am. Chem. Soc. 1988, 110, 1069-1074.

44 C. E. Tait, P. Neuhaus, H. L. Anderson, C. R. Timmel, J. Am. Chem. Soc. 2015, 137, 6670-6679. 
45 P. A. Jänne, N. Gray, J. Settleman, Nature Reviews Drug Discovery. 2009, 8 (9) 709-23.

46 N. P. Shah, C. Tran, F. Y. Lee, P. Chen, D. Norris, C. L. Sawyers, Science 2004, 305, 399-401.

47 E. Weisberg, P. W. Manley, S. W. Cowan-Jacob, A. Hochhaus, J. D. Griffin, Nat. Rev. Cancer 2007, 7, 345-356.

48 J. S. Tokarski, et al. Cancer Res. 2006, 66, 5790-5797. www.rcsb.org/structure/2GQG

49 T. Ma et al., Science, 2018, 361, 6397, 48-52.

50 Y. Wang, P. Pigeon, S. Top, J. S. Garcia, C. Troufflard, I. Ciofini, M. J. McGlinchey, G. Jaouen, Angew. Chem. Int. Ed. 2019, 58, $8421-8425$. 
Supplementary information for:

\section{Biradical formation by proton extraction in thiazole derivatives:}

\section{The hidden nature of Dasatinib.}

Carlos Heras, ${ }^{1}$ Daniel Reta, ${ }^{2 *}$ Rosendo Valero ${ }^{3,4}$ Guillem Albareda, ${ }^{4}$ Nicholas F. Chilton, ${ }^{2}$ Alistair Fielding, ${ }^{5}$ Ibério de P. R. Moreira, ${ }^{3,4 *}$ Josep Maria Bofill, ${ }^{1,4}$ and Francisco López-Calahorra, ${ }^{1}$ ${ }^{1}$ Departament de Química Inorgànica i Orgànica, Sec. de Química Orgànica, Universitat de Barcelona, C/ Martí i Franquès 1, E-08028 Barcelona, Spain. ${ }^{2}$ School of Chemistry, The University of Manchester, Oxford Road, Manchester, M13 9PL, United Kingdom.

${ }^{3}$ Departament de Ciència dels Materials i Química Física, Sec. de Química Física, Universitat de Barcelona, C/ Martí i Franquès 1, E-08028 Barcelona, Spain.

${ }^{4}$ Institut de Química Teòrica i Computacional (IQTCUB), Universitat de Barcelona, C/ Martí i Franquès 1, E-08028 Barcelona, Spain.

${ }^{5}$ School of Pharmacy and Biomolecular Sciences, Liverpool John Moores University, Byrom Street, Liverpool, L3 3AF, United Kingdom..

Contents:

1. Synthesis of compounds 1-3 and $\mathbf{5}$ and purchase of molecule $\mathbf{4}$.

2. Characterisation techniques.

2.1. EPR spectroscopy and SQUID magnetometry.

2.1.1. Continuous-wave EPR.

2.1.2. Pulse-EPR.

2.1.3. SQUID.

2.2. UV-Vis.

2.3. NMR.

3. A valence bond topological interpretation of the biradical species.

4. Modelling the electronic structure: CASSCF and CASCI calculations on $\mathbf{1 .}$

5. References. 


\section{Synthesis of compounds 1-3 and purchase of molecule 4.}

All reagents and solvents used in the synthesis described below are high-grade commercial products and have been used without further purification. NMR experiments for compounds 1-4 were performed in d6-DMSO (using residual DMSO as reference) and $\mathrm{CDCl}_{3}$ (using TMS as a internal reference) at Varian Mercury $400 \mathrm{MHz}$ spectrometer. IR spectra were acquired using a Nicolet 6700 FT-IR spectrometer; wave numbers of characteristic peaks are reported in $\mathrm{cm}^{-1}$. High resolution and chemical ionization mass spectra were obtained using an Agilent Technologies LC/MSD TOF spectrometer and a ThermoFinnigan TRACE DSQ spectrometer, respectively, both facilities available in the Serveis Cientifico-Tècnics (SCT) of the University of Barcelona.

Compound 1: (2,6-dimethyl-4-nitrophenyl)thiazol-2-amine)

Synthesis and characterization of (2,6-dimethyl-4-nitrophenyl)-thiourea (compound $\mathbf{1 b}$ )

Compound 1a was previously synthetized following a procedure described in reference $\left.{ }^{1}\right]$ )

Figure S 1. Synthesis of compound $\mathbf{1 .}$

Potassium thiocyanate $(1,23 \mathrm{mmol})$ and benzoyl chloride $(1,20 \mathrm{mmol})$ were dissolved in acetone. This solution was heated at $56^{\circ} \mathrm{C}$ during 1 hour. Then, a solution of compound 1a $(1,20 \mathrm{mmol})$ in acetone $(10 \mathrm{ml})$ was added to the initial solution and was heated at the same temperature during 2 hours. Crude solution was cooled to r.t. and water $(50 \mathrm{ml})$ was added. Then, the crude mixture was extracted with $25 \mathrm{ml}$ ethyl acetate and the resulting organic layer was separated and dried with anhydrous magnesium sulphate. The solvent was removed by distillation and $15 \mathrm{ml}$ of sodium hydroxide solution (10\%) were added to the resulting crude dissolved in methanol $(10 \mathrm{ml})$. The mixture was boiled 
gently under reflux for 30 min and cooled to r.t. Then, $\mathrm{HCl}$ (5\%) solution was added until acid $\mathrm{pH}$ and $\mathrm{NaHCO}_{3}$ saturated solution until $\mathrm{pH}$ 7. At this moment, a yellow precipitate of $\mathbf{1 b}$ was obtained. The solid was isolated by filtration. Yield $65 \%$. 1H NMR (400 MHz, DMSO-d6): $\delta(p p m)=9.27(1 \mathrm{H}, \mathrm{s}$, b.s.), 7.99 (2 H, s), 2.28 (6 H, s). 13C NMR (100 MHz, $\left.\mathrm{CDCl}_{3}\right): \delta(\mathrm{ppm})=181.91(\mathrm{C}), 170.28(\mathrm{C})$, 145.62 (C), 138.53 (C), 129.18 (C), 122.55, 17.88 (2CH3). FTIR (KBr): v $\left(\mathrm{cm}^{-1}\right)=3385,3290,3196$, 3128, 2923, 1631, 1595, 1513, 1439, 1344, 1290, 1216, 1101, 1071, 1035, 941, 898, 854, 807, 746, 711, 649. (HRMS-ESI(+)) calcd. for C9H12N3O2S: 225.0572, found 225.0572. The solid was used in the next step without purification.

Synthesis and characterization of (2,6-dimethyl-4-nitrophenyl)thiazol-2-yl-amine (compound 1) 4,00 mmol of compound $\mathbf{1 b}$ were dissolved in $25 \mathrm{ml}$ of acetone. An aqueous chloroacetaldehyde $45 \%$ solution (1,17 mL, 2 eq) was added to the previous one and the mixture was refluxed for $16 \mathrm{~h}$. After that, the solvent was removed by distillation and re-dissolved in dichloromethane. The organic solution was discarded. Sodium hydroxide $10 \%$ water solution was used to neutralize the aqueous layer, which was extracted with ethyl acetate. The solvent was removed and the resultant crude was digested in a mixture of ether and acetone. A yellow solid $\mathbf{1}$ was obtained and isolated by filtration. Yield $43 \%$.

FT-IR $(\mathrm{KBr}): v\left(\mathrm{~cm}^{-1}\right)=3376,3116,3066,2922,2851,2762,1708,1601,1561,1508,1467,1448$, 1417, 1336, 1207, 1160, 1099, 1072, 1034, 943, 897, 843, 783, 771, 744, 715, 598.

${ }^{1}$ H NMR (400 MHz, DMSO-d6): $\delta$ (ppm) =9.51 (bs, 1 H), 8.02 (s, 2 H), 7.09 (d, 4 Hz, 1H), 6.82 (1 H, bd, $4 \mathrm{~Hz}), 2.27$ (6 H, s).

${ }^{13}$ C NMR (100 MHz, CDCl3): $\delta(p p m)=146.35$ (C), 142.81 (C), 137.47 (C), 137.33 (CH), 135.25 (C), $124.00(\mathrm{CH}), 107.78(\mathrm{CH}), 18.53$ (2CH3).

HRMS-ESI(+): calcd. for C11H11N3O2S: 249.0572, found 249.0572.

The absence of traces of the most common magnetic metals has been established by plasma emission spectrometry. The amount of Fe, Co and Ni traces of the samples of compound 2 obtained is close to the limit of detection of the technique with respect to the blank solvent (5.2 ppm, $1.2 \mathrm{ppm}$ and 0.2 ppm, respectively).

Compound 2: N-(2-nitroacridin-6-yl)-2-aminobenzothiazole

Synthesis and characterization of compound $\mathbf{2}$ was performed as described in reference $\left[{ }^{2}\right]$.

Compound 3: N-methyl-N-(2-nitroacridin-6-yl)-2-imino benzothiazole

Synthesis and characterization of compound 3 was performed as described in reference $\left[{ }^{3}\right]$.

Compound 4: N-(2-chloro-6-methylphenyl)-2-((6-(4-(2-hydroxyethyl)piperazin-1-yl)2-methylpyrimidin-4-yl)amino)thiazole-5-carboxamide (Dasatinib) 
Commercial sample sold by Seleckchem (99.76\% purity by HPLC). NMR spectra consistent with structure.

Compound 5: N-(4-nitrophenyl)-9H-fluoren-9-imine

Synthesis and characterization of N-(4-nitrophenyl)-9H-fluoren-9-imine (compound 5) has been performed in order to have a diamagnetic molecule that has been synthesised using similar procedures and reagents/solvents as in the previous case. It is used here as a blank test for EPR and the topological VB model (see Sect 3. The synthesis of compound $\mathbf{5}$ is a one-step reaction followed by purification similar to previously reported procedures $\left[{ }^{4}-5\right]$ :

Figure S 2. Synthesis of compound 5.

In this case, 2,00 g of 9H-fluoren-9-one (1 eq, 11,0 mmol) with 2,52 $\mathrm{g}$ of $\mathrm{AlCl}_{3}(1,7 \mathrm{eq}, 18,8 \mathrm{mmol})$ were dissolved in $50 \mathrm{~mL}$ of $\mathrm{CHCl}_{3}$ and stirred during 10 minutes. After this time, 3,07 $\mathrm{g}$ of 4nitroaniline (2 eq, 22,2 mmol) and $8 \mathrm{~mL}$ of $\mathrm{NEt}_{3}$ (5,2 eq, 57,4 mmol) were added and the mixture were stirred during $18 \mathrm{~h}$ at ambient temperature. Then, the organic phase was washed using $3 \times 30 \mathrm{~mL}$ of $5 \% \mathrm{HCl}$ in $\mathrm{H}_{2} \mathrm{O}$. The organic phases were dried with $\mathrm{MgSO}_{4}$ anh and the solvent was evaporated at reduced pressure. The resultant crude was purified by means of column cromatography on silica gel using DCM as eluent. CCF: Rf = 0,75, eluent AcOEt. Finally, 3,73 g of a yellow solid $\mathbf{5}$ were obtained,. Yield: $70 \%$.

IR $\left(\mathrm{KBr} \mathrm{cm}^{-1}\right)$ : 3090, 1661, 1599, 1571, 1559, 1540, 1522, 1466, 1449, 1348, 1302, 1273, 1244, 1210 , 1194, 1160, 1145, 1101, 1090, 1075, 987, 946 912, 885, 820, 801, 791, 766, 744, 731, 720, 694, 672, 651, 617, 595, 550.

${ }^{1} \mathbf{H}$ NMR (400 MHz, CDCl 3 , ppm): 8,07 (1H, dd, $\left.J=8,0 \mathrm{~Hz}, J=2,0 \mathrm{~Hz}\right), 7,89(1 \mathrm{H}, \mathrm{d}, J=7,2 \mathrm{~Hz})$, 7,87 (1H, d, $J=2,0 \mathrm{~Hz}), 7,62$ (1H, dd, $J=8,0 \mathrm{~Hz}, J=2,8 \mathrm{~Hz}), 7,57(1 \mathrm{H}, \mathrm{d}, J=8,0 \mathrm{~Hz}), 7,50(1 \mathrm{H}, \mathrm{t}, J$ $=7,2 \mathrm{~Hz}), 7,37(2 \mathrm{H}, \mathrm{tm}, J=7,6 \mathrm{~Hz}), 7,33(1 \mathrm{H}, \mathrm{dm}, J=8,0 \mathrm{~Hz}), 6,93(1 \mathrm{H}, \mathrm{t}, J=7,6 \mathrm{~Hz}), 6,51(1 \mathrm{H}, \mathrm{d}$, $J=8,0 \mathrm{~Hz})$.

${ }^{13}$ C NMR (400 MHz, $\mathrm{CDCl}_{3}$, ppm): 164,2 Cq, 152,7 Cq, 149,1 Cq, 144,2 Cq, 142,0 Cq, 136,9 Cq, 132,5, 130,8 Cq, 130,2, 128,7, 127,8, 126,7, 124,7, 123,6, 120,7, 119,8, 118,7, 113,6.

High resolution ESI: Found $[\mathrm{M}+\mathrm{H}]^{+}=301,0973$, calculated for $\left(\mathrm{C}_{19} \mathrm{H}_{12} \mathrm{~N}_{2} \mathrm{O}_{2}\right) 301,0977$. 


\section{Characterisation techniques.}

2.1. $\quad$ EPR spectroscopy and SQUID magnetometry.

\subsubsection{Continuous-wave EPR.}

X-band spectra were recorded with a Bruker EMX-micro spectrometer fitted with a Super High Q X-band resonator. Polycrystalline samples were placed in $4 \mathrm{~mm}$ quartz X-band EPR tubes. The tubes were de-gassed with helium to remove traces of oxygen. Powder of molecule 1 was dissolved in DCM, whereas molecules 2-4 were dissolved in DMSO due to solubility problems in DCM. Addition of the base (DBU) and subsequent addition of acetic acid was performed under a positive flow of helium directly into the X-band tube through the sealing rubber cap, to avoid possible signal loss by interaction with molecular oxygen. All spectra have been collected with 4 Gauss modulation and $30 \mathrm{~dB}$ attenuation, unless specified otherwise.
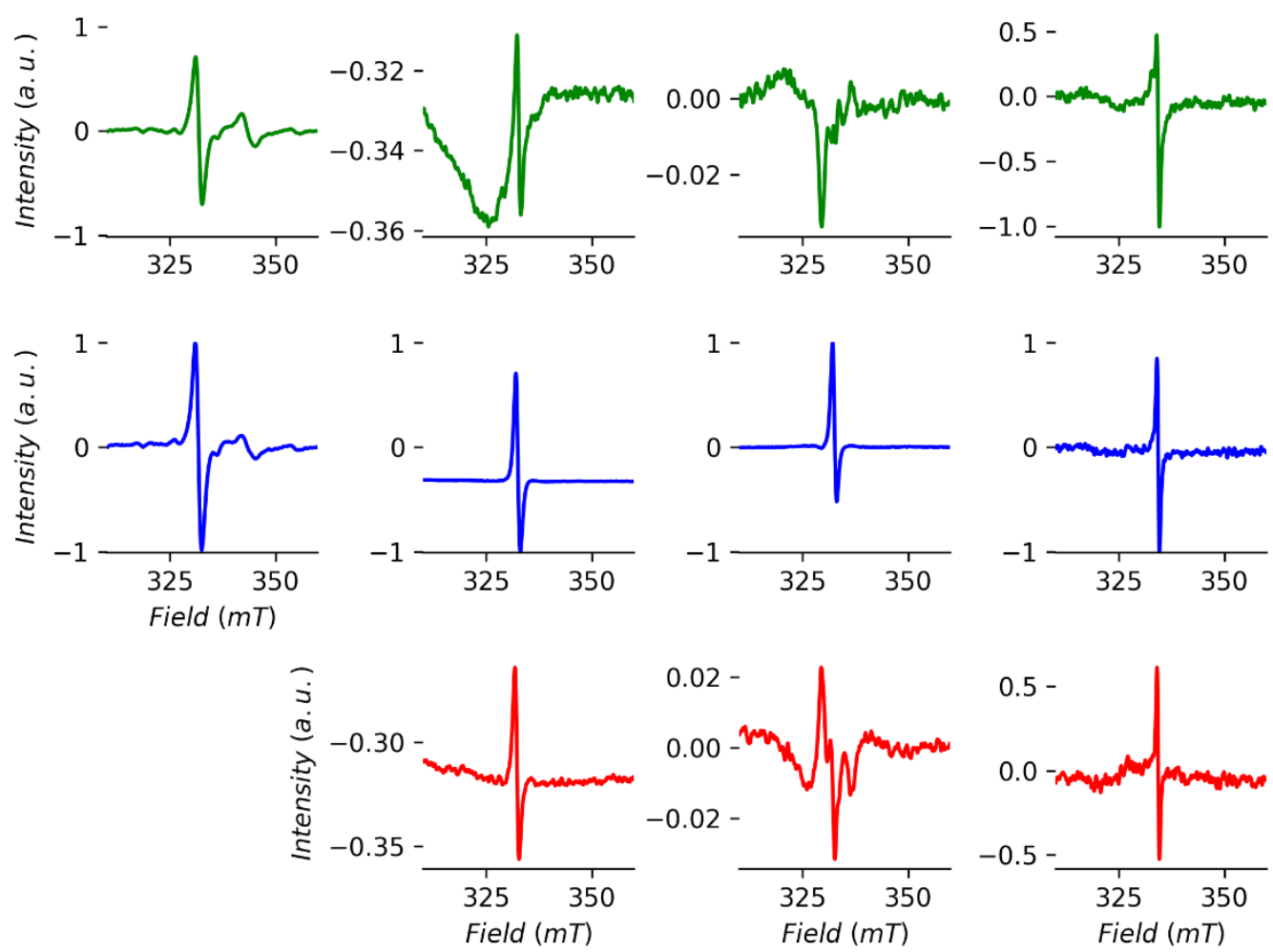

Figure S 3. Comparison of X-band EPR spectra of 1-4 frozen solutions (12.5 K for 1, $5 \mathrm{~K}$ for 2-4). Columns 1 to 4 indicate molecules 1-4, respectively. Top, middle and bottom row indicate the neutral, basic and acidic solutions, respectively. 

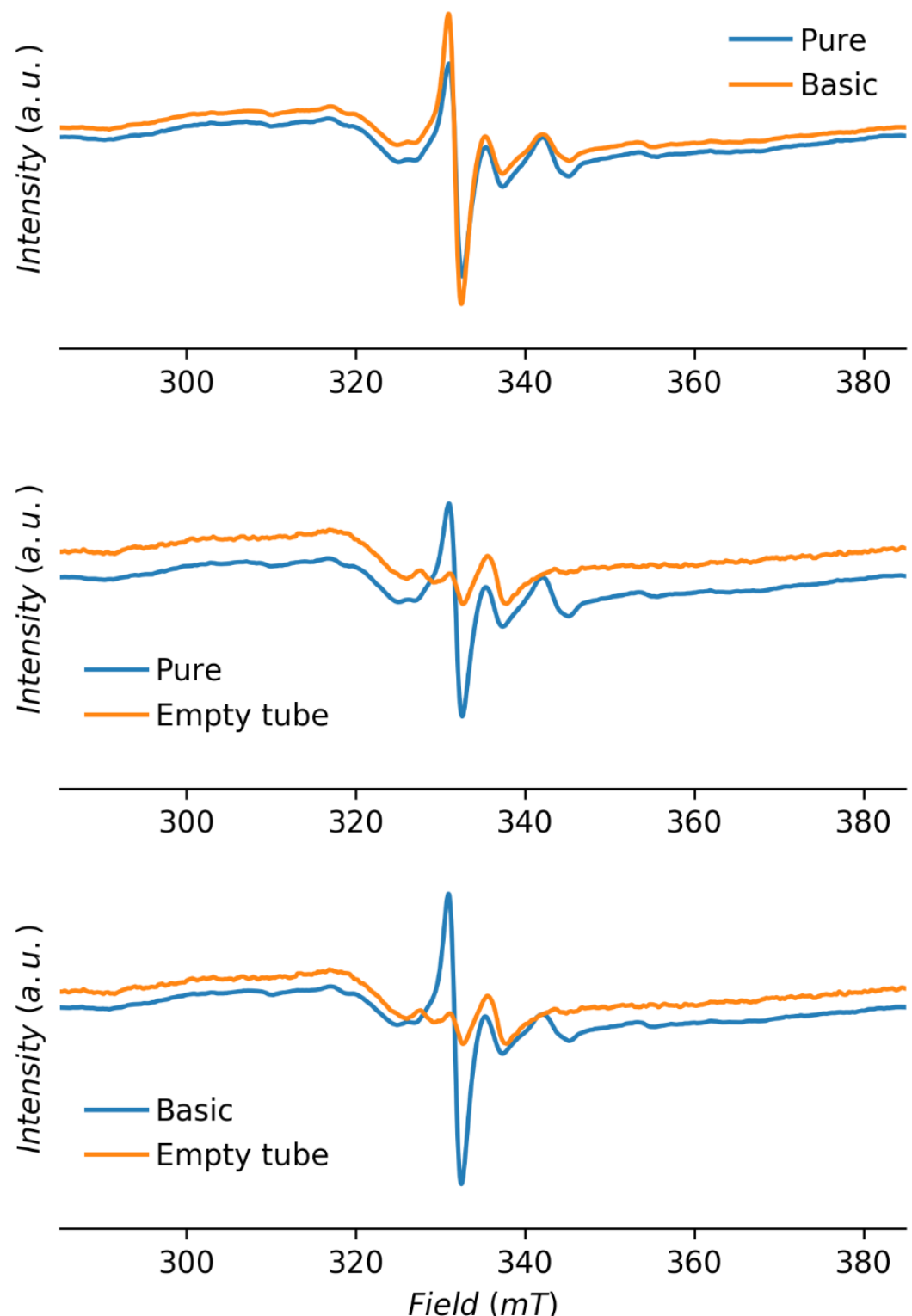

Figure S 4. Comparison of X-band EPR spectra of $\mathbf{1}$ frozen solutions and empty tube at $12.5 \mathrm{~K}$. Due to the weakness of signal, background subtraction results in artificial features (see Figure 1 in main text). 


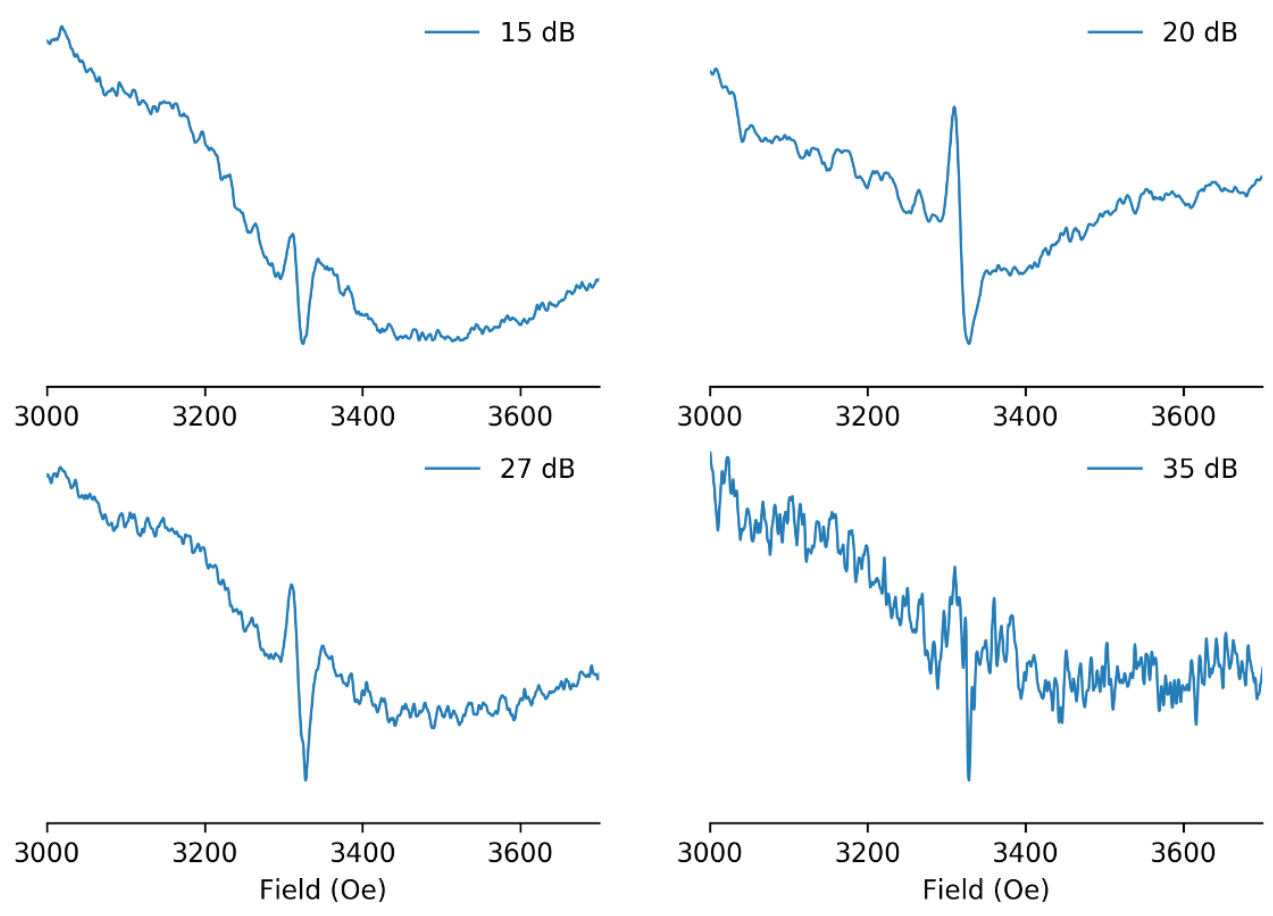

Figure S 5. Power dependence of X-band EPR spectra of powder 4 at $295 \mathrm{~K}$, measured with 7, 2.2, 0.4 and $0.07 \mathrm{~mW}$, respectively.

The synthesis of $\mathbf{5}$ was performed following the exact same procedure as for compounds 1-3 (see Section SI 1).

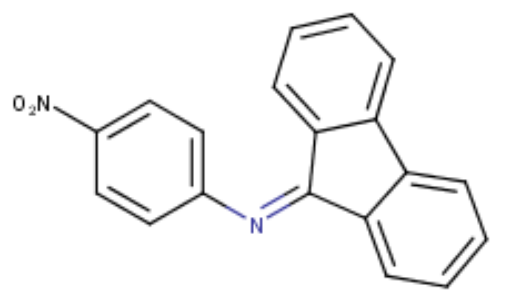

Figure S 6. Molecular structure of compound 5, used as a control.

Compound $\mathbf{5}$ is used here as a counterexample to show that acidic $\mathrm{H}$ atoms are required to generate the paramagnetic species responsible of the observed EPR response. In addition, the fact that the synthesis of this compound uses similar procedures and uses the same solvents and similar reactants, ensures the absence of impurities, in particular paramagnetic ions. We provide also the blank lines of the tube containg only solvent and DBU. 

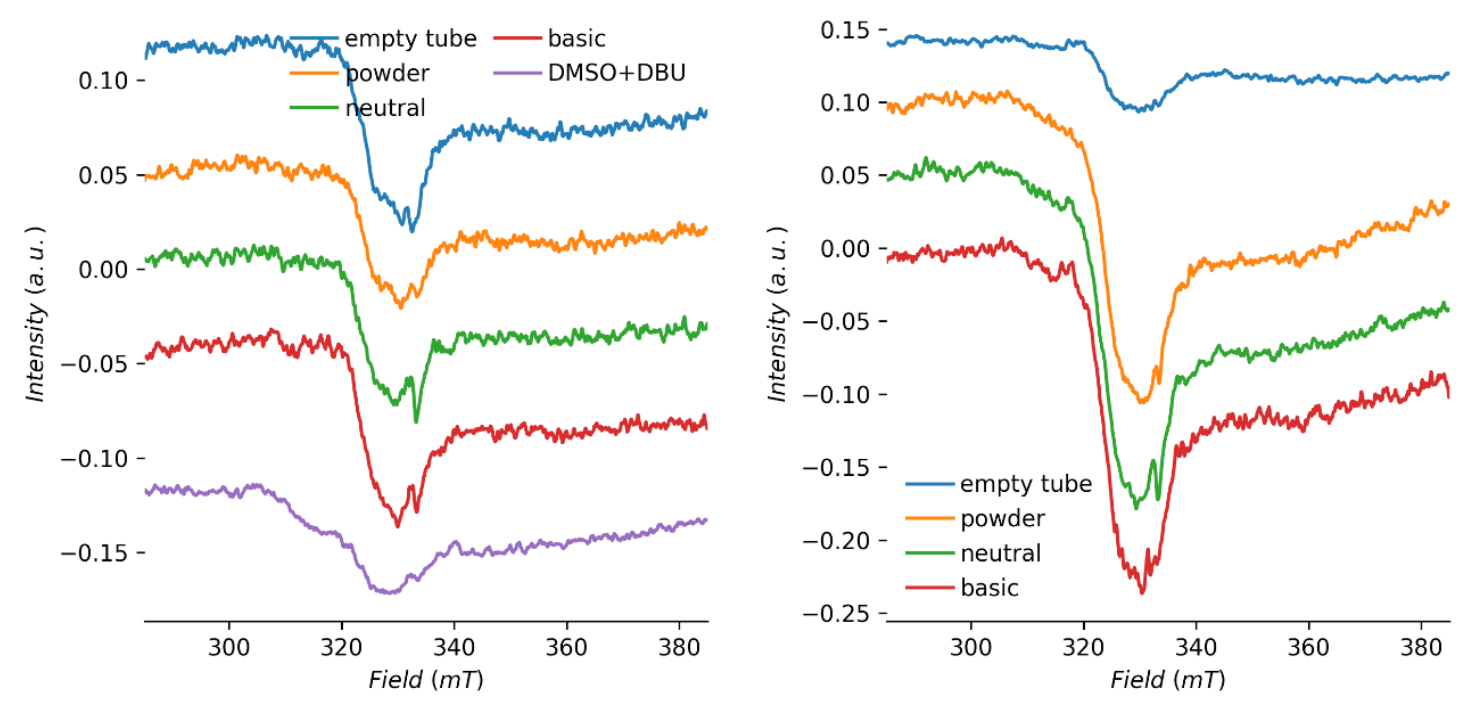

Figure S 7. Powder and frozen solution X-band EPR spectra of molecule 5 at $5 \mathrm{~K}$, measured with 0.22 (left) and 2.2 (right) $\mathrm{mW}$ power. The empty tube and a mixture of DMSO and DBU base is also shown at $8 \mathrm{~K}$.

EPR spectra are simulated with PHI. ${ }^{6}$ The model spin Hamiltonian employed is defined as

$$
\widehat{H}=-2 \sum_{i<j}^{i, j \in N} \hat{S}_{i} \cdot \dot{J}_{i j} \cdot \hat{S}_{j}+\mu_{B} \sum_{i=1}^{N} \hat{S}_{i} \cdot g_{l} \cdot \vec{B}
$$

where the exchange and g matrices are assumed isotropic. For the case of a spin doublet ( $S=1 / 2$ ), only the Zeeman part is considered. Figure $S 8$ presents the X-band powder and solution spectra (after addition of the DBU base) of molecule 3 recorded at $5 \mathrm{~K}$, compared to a simulation assuming two centres interacting very weakly ferromagnetically and a single centre. It is worth noting that the ratio between the g-values and the magnetic exchange constant $\mathrm{J}$ are the same for the simulated data in powder and solution. The fact that assuming a single or two unpaired electrons in our spin Hamiltonian provides equally good simulations of the EPR data requires additional arguments to discern between the two. In order to do that we resort to UV-Vis and NMR spectra (see below) to gain chemical insight into why the whole set of results only make sense if we have a molecular spin triplet, rather than a spin doublet as paramagnetic species in the samples. 


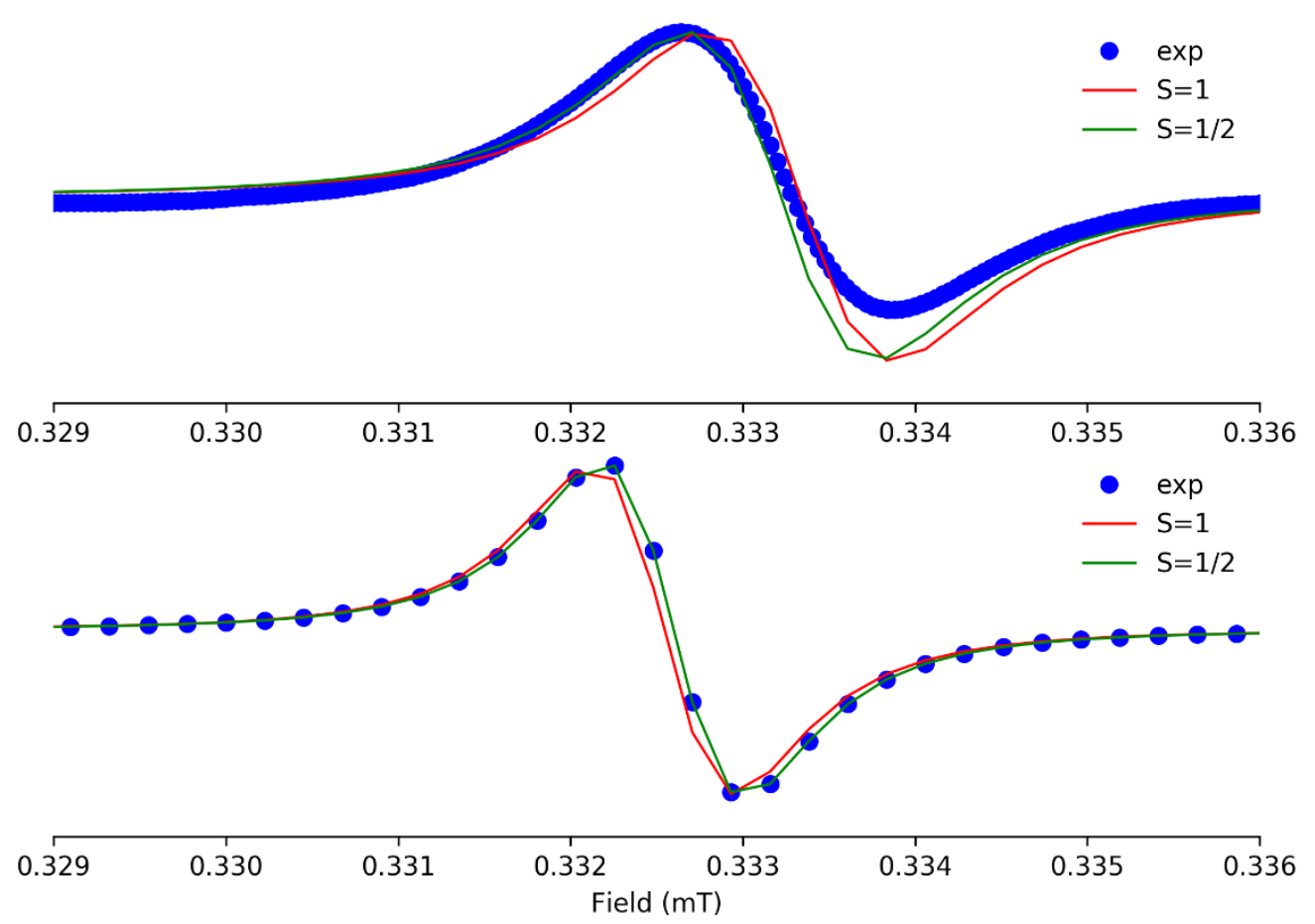

Figure S 8. Comparison of EPR spectrum (circles) for molecule 3 at $5 \mathrm{~K}$ and the results of assuming a model spin Hamiltonian with two unpaired electrons (red line, $S=1$ ) and one unpaired electron

(green line, $S=1 / 2$ ). Top and bottom figures refer to powder and solution data. Parameters employed in the simulation are presented in Table $\mathbf{S} \mathbf{1 .}$

According to our proposed model, the delocalised unpaired electrons are mostly based on nitrogen centres and therefore one would expect to observe the hyperfine coupling to the $I=1$ nuclear spin of ${ }^{14} \mathrm{~N}$ in continuous wave EPR spectra. Figure S 9 shows how with a sensible set of parameters, one can mask the hyperfine and end up with an isotropic spectrum as the ones we have recorded. We use PHI to simulate the spectra and this time we include an extra term to the Hamiltonian accounting for the nuclear-spin interaction:

$$
\widehat{H}=-2 \sum_{i<j}^{i, j \in N} \hat{S}_{i} \cdot \dot{J}_{i j} \cdot \hat{S}_{j}-2 \sum_{i<j}^{i, j \in N} \hat{I}_{i} \cdot \dot{A}_{i j} \cdot \hat{S}_{j}+\mu_{B} \sum_{i=1}^{N} \hat{S}_{i} \cdot \dot{g}_{l} \cdot \vec{B}
$$

Table S 1. Summary of parameters used in the model spin hamiltonians to simulate the EPR spectra of molecule 3 .

\begin{tabular}{l|cc|cc}
\multicolumn{1}{c}{} & \multicolumn{2}{c|}{ Solid } & \multicolumn{2}{c}{ Solution } \\
\cline { 2 - 5 }$g_{1}$ & triplet & doublet & triplet & doublet \\
\cline { 2 - 5 }$g_{2}$ & 2.0105 & 2.0103 & 2.0143 & 2.0133 \\
$l w$ & 2.0091 & & 2.0129 & \\
$J_{12}$ & 0.0494 & 0.0494 & 0.0359 & 0.0357 \\
$g_{1}$ & 0.0010 & & 0.0014 & \\
$g_{2}$ & 1.00069 & & 1.00069 &
\end{tabular}


The hyperfine coupling constants used in the simulation are consistent with a similar diradical $^{7}$ that displays nitrogen-based delocalised electrons. The rest of the parameters entering the Hamiltonian are consistent with those used to simulate the spectrum of molecule 3.

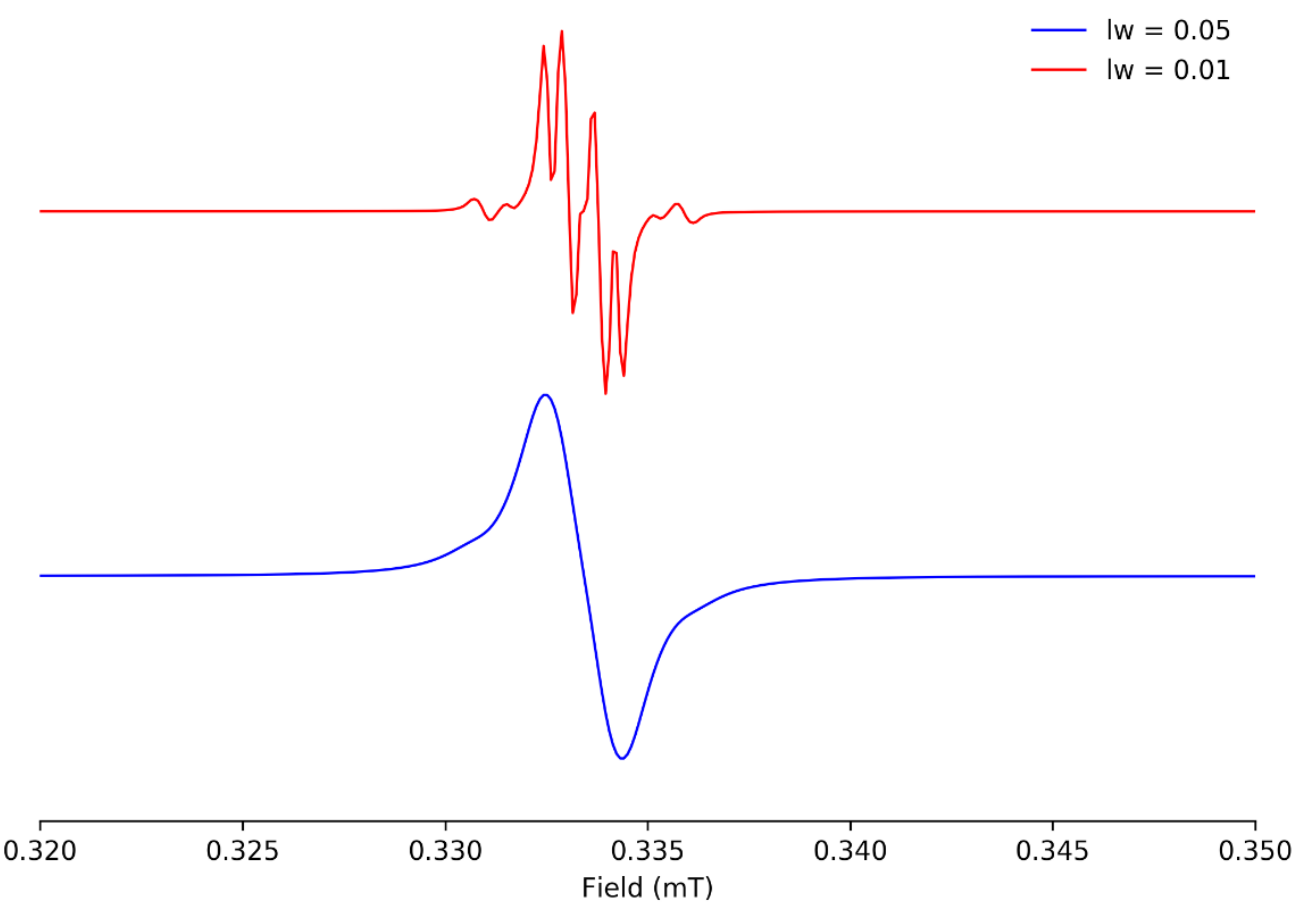

Figure S 9. Resolving hyperfine coupling. Both spectra have been simulated with $g_{1}=2.0105$, $g_{2}=2.0091, A=0.0004 \mathrm{~cm}^{-1}$ and $J_{12}=0.001 \mathrm{~cm}^{-1}$, with a varying linewidth. Blue line uses a linewidth in line with the experimental values. 


\subsubsection{Pulse-EPR.}

Pulse-EPR experiments were carried out using an X-band Bruker Elexsys E580 spectrometer with an Oxford CF 935 cryostat. The echo detected field sweep spectra of 2 and 3 are presented in Figure S 10 and Figure S 11. Echo detected field swept EPR spectra were collected using a two pulse (16 and 32 ns) Hahn-echo sequence.

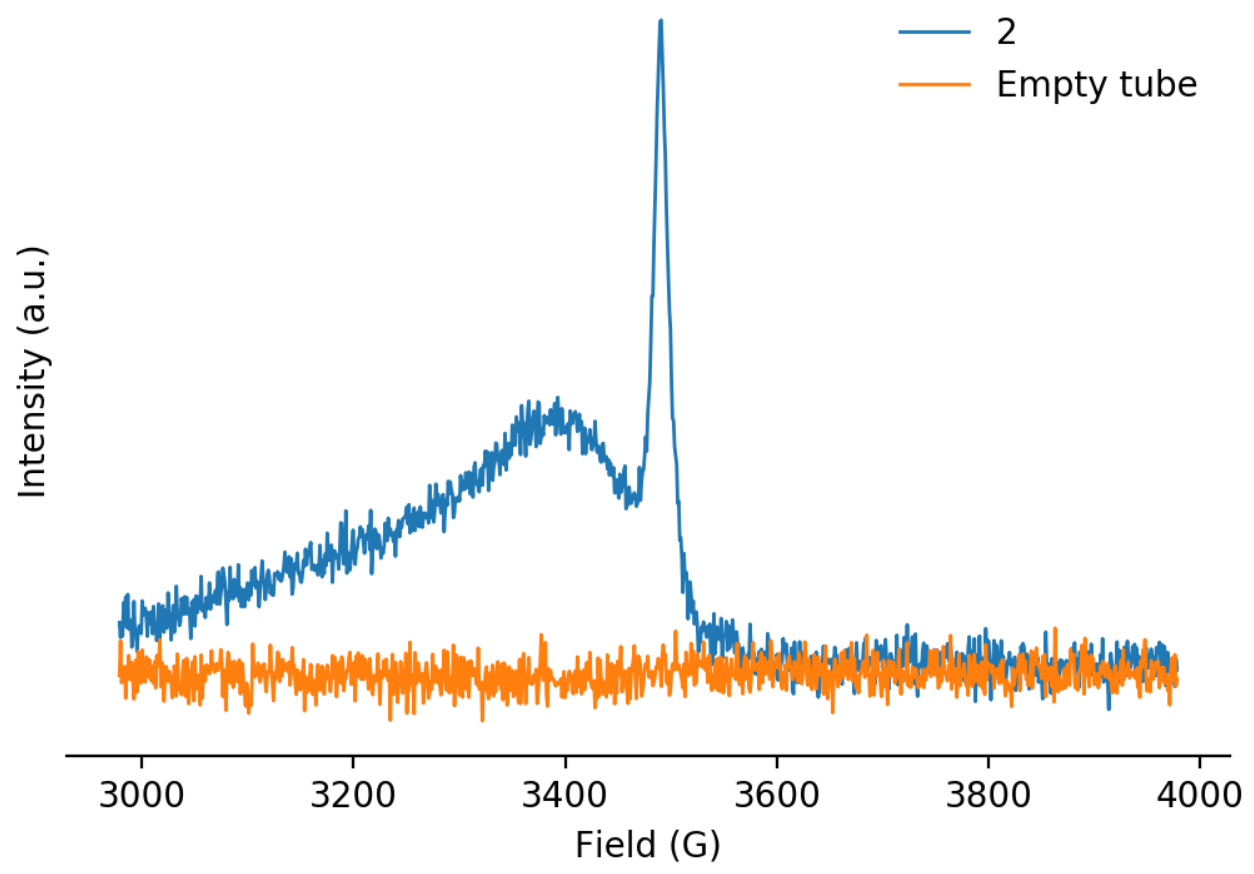

Figure S 10. X-band echo detected field-sweep spectra, comparison of powder $\mathbf{2}$ and empty tube at 5 K.

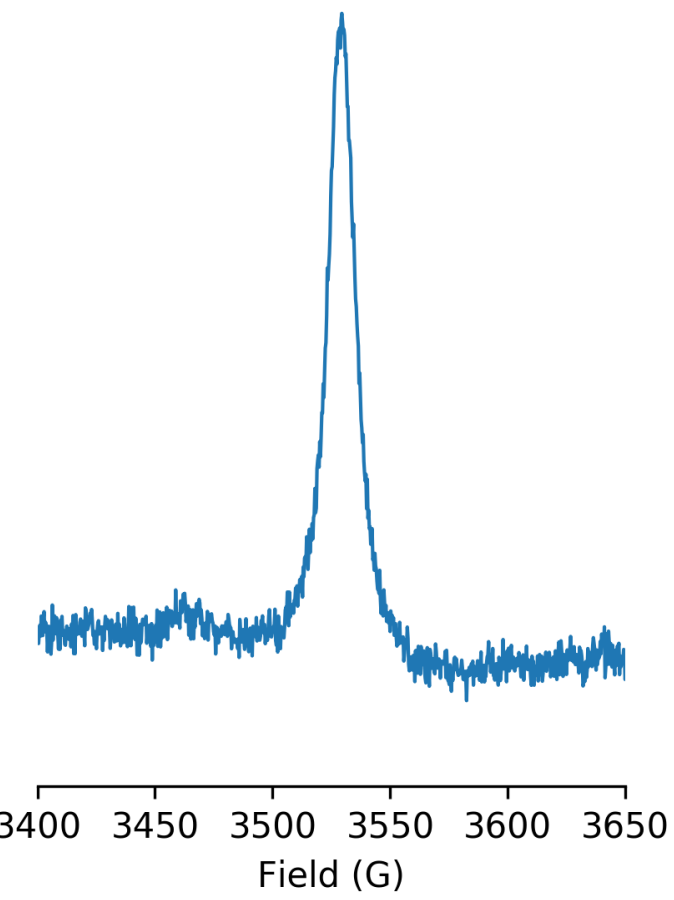

Figure S 11. X-band echo detected field-sweep spectrum of powder 3 at $5 \mathrm{~K}$. 


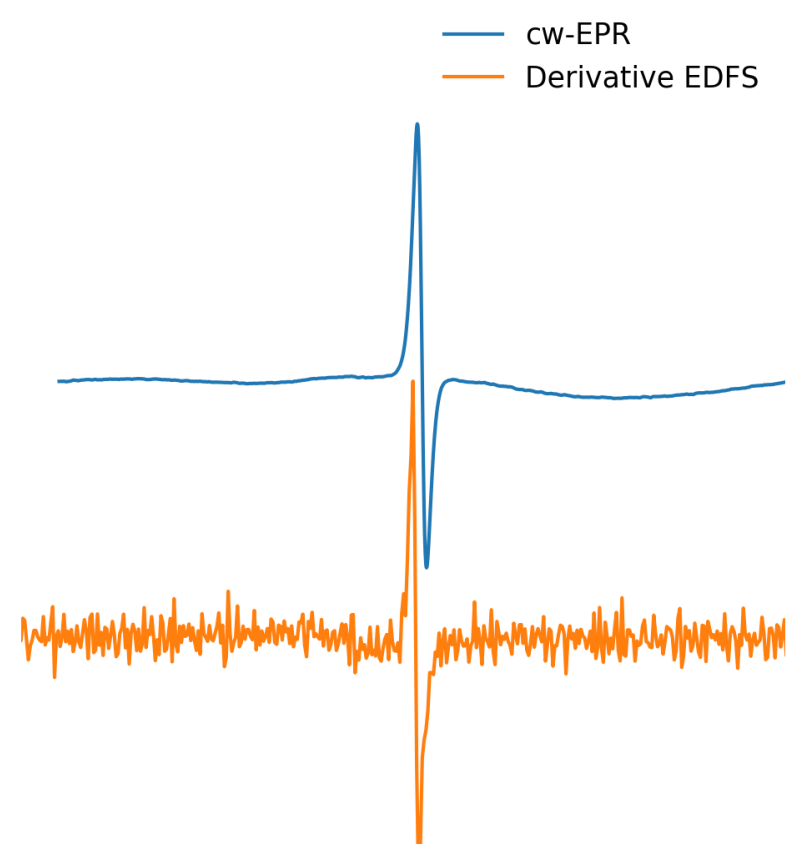

Figure S 12. Comparison of powder cw-EPR and derivative of the Echo Detected Field Sweep spectra of 2 at $5 \mathrm{~K}$.

To address the presence of hyperfine interaction, we apply two- ( $\pi / 2-\tau-\pi-\tau-e c h o)$ and three-pulse $(\pi / 2-\tau-\pi / 2-T-\pi / 2-\tau-e c h o)$ sequences to record the electron spin echo envelope modulation (ESEEM) due to the presence of ${ }^{14} \mathrm{~N}$ nuclear spins. Taking the Fourier transform of the echo's envelope in the time domain one obtains the frequency domain ESEEM results, which allows access of the nuclear quadrupole resonances (Figure S 13, Figure S 14 and Figure S 16). Time-domain data of the 3-pulse ESEEM on 2 presents an abrupt decay at the beginning of the collection, most likely due to an incorrect allignement of the pulses and the trigger. Still, its Fourier Transform presents clear peaks at low frequency, typical of ${ }^{14} \mathrm{~N}$ and in accordance with the 2-pulse data. 

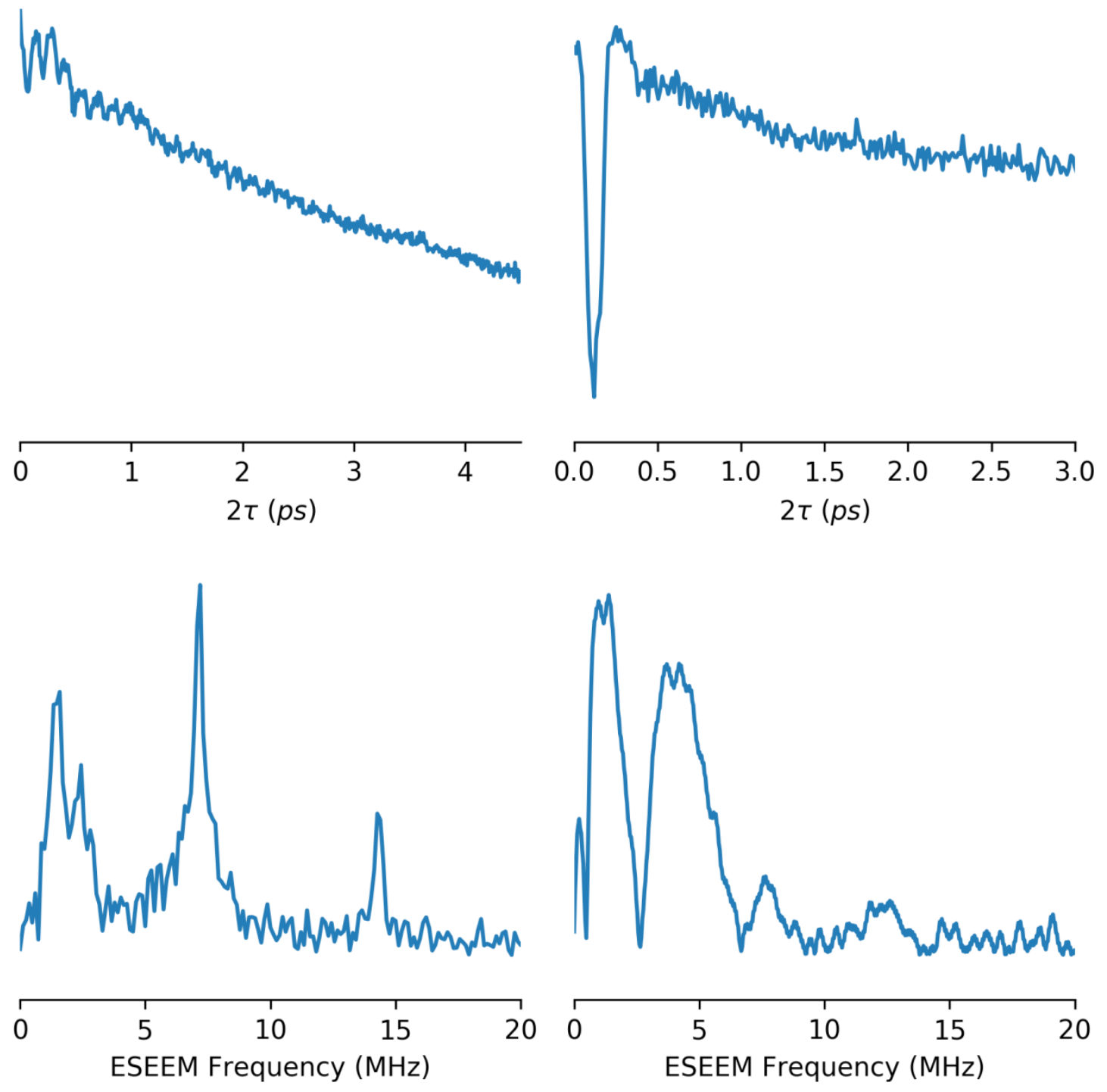

Figure S 13. X-band pulse EPR spectra of molecule 2 at $5 \mathrm{~K}$ at $\mathrm{H}_{\mathrm{dc}}=3454 \mathrm{G}$ field position. Top and bottom rows indicate the time- and frequency-domain of the ESEEM, respectively. Left and right columns present the data for 2- and 3-pulses. 

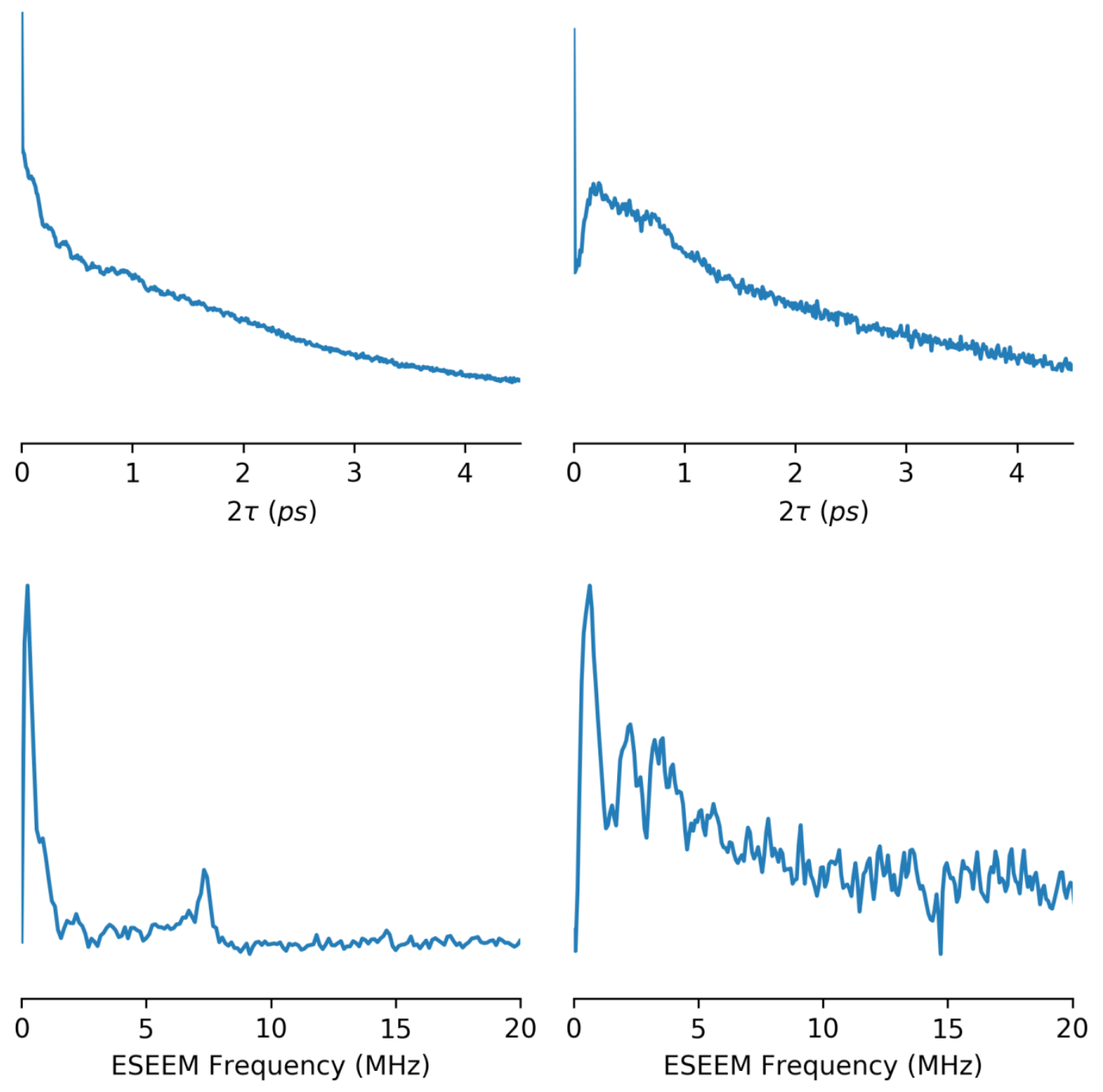

Figure S 14. X-band pulse EPR spectra of molecule 2 at $5 \mathrm{~K}$ at $\mathrm{H}_{\mathrm{dc}}=3550 \mathrm{G}$ field position. Top and bottom rows indicate the time- and frequency-domain of the ESEEM, respectively. Left and right columns present the data for 2- and 3-pulses. 

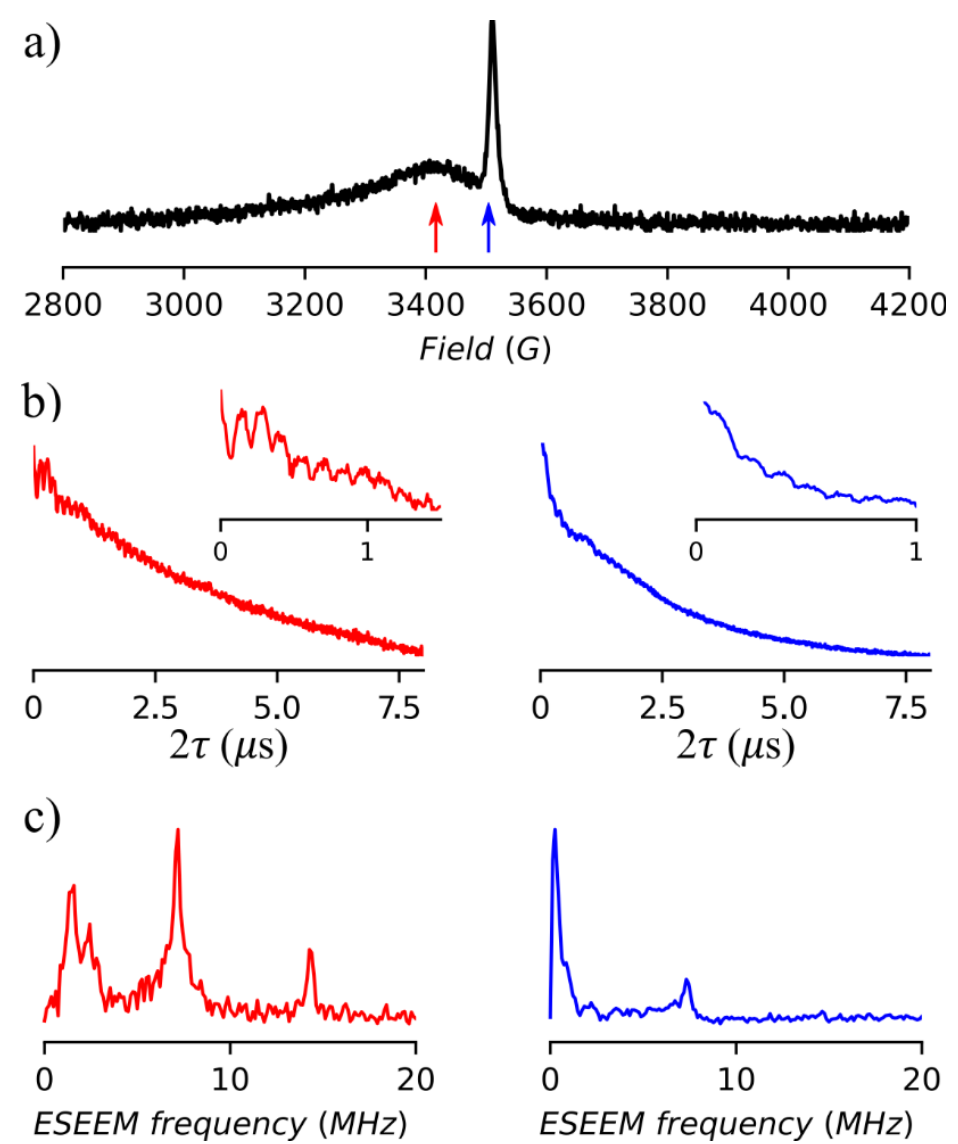

Figure S 15. Summary of X-band 2-pulse EPR spectra of molecule 2 at 5 K. a) Echo detected fieldsweep spectrum. b) Time-domain of 2-pulse electron spin echo envelope modulation (ESEEM). c) Frequency domain (Fourier transform) of the T2 experiment. 

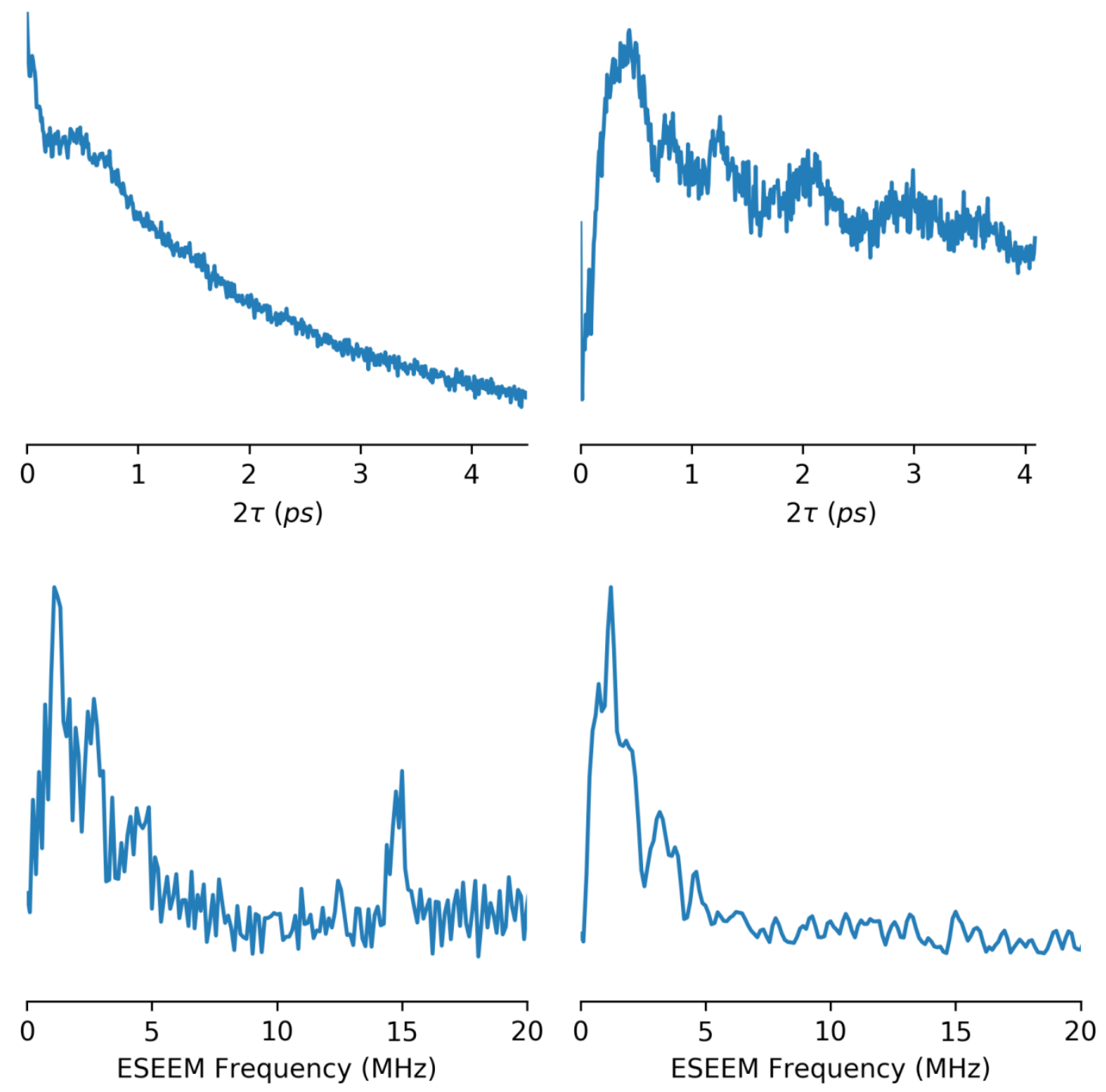

Figure S 16. X-band pulse EPR spectrum of molecule 3 at $5 \mathrm{~K}$. Top and bottom rows indicate the time- and frequency-domain of the ESEEM, respectively. Left and right columns present the data for 2- and 3-pulses.

3-pulse data on 3 shows a simpler frequency profile, which potentially can be modelled. The nuclear quadrupole interaction can be described as a traceless matrix encoding the interaction of a nuclear quadrupole moment with the electric field gradient of the surrounding electrons. Its matrix representation is

$$
\widehat{H}_{N Q I} / h=\hat{I}^{T} Q \hat{I}=\sum_{i=x y z} Q_{i j} \hat{I}_{i} \hat{I}_{j}
$$

where

$$
Q=\frac{e^{2} Q q / h}{4 I(2 I-1)}\left(\begin{array}{ccc}
-(1-\eta) & 0 & 0 \\
0 & -(1+\eta) & 0 \\
0 & 0 & 2
\end{array}\right)
$$


with the anisotropic parameter $\quad \eta=\frac{Q_{11}-Q_{22}}{Q_{33}} \quad$ and $\quad K=\frac{e^{2} Q q / h}{4 I(2 I-1)}=\frac{e^{2} Q q}{4 h} \quad$ for $I=1$. Fortunately, for molecule 3 using a 3-pulse sequence we observe a clear relationship between the measured resonances, which indicates that we are near the cancellation conditions with the anisotropic tensor twice as big as the Larmor frequency of the nuclear spin ( $v_{I} \cong A / 2$ ). Under these conditions, the effective field experienced by an $I=1$ nucleus vanishes in one of the two $m_{s}$ manifold and the nuclear frequencies within this manifold correspond to the nuclear quadrupole resonance(NQR) frequencies ${ }^{8}$

$$
\begin{gathered}
v_{0}=2 K \eta \\
+i=\frac{K(3+\eta)}{v_{i}} \\
-i=K(3-\eta) \\
v_{i}
\end{gathered}
$$

with $\begin{gathered}-i \\ +i=v_{0}+v_{i} \\ v_{i}\end{gathered}$. From Figure S 17, one can assign $\begin{gathered}+i=1.829 \\ v_{i}\end{gathered}, \begin{gathered}-i=1.219 \\ v_{i}\end{gathered}$ and $v_{0}=0.732 \mathrm{MHz}$, which translate into $K=0.528 \mathrm{MHz}$ and $\eta=0.693$. Using these values as a starting point, the 3-pulse ESEEM was fitted using EasySpin ${ }^{9,10}$ and the built-in 'saffron' function, employing a single $\mathrm{S}=1 / 2$ and a ${ }^{14} \mathrm{~N}$ centre defined as:

$$
\widehat{H}=\mu_{B} B^{T} g \hat{S}-\mu_{n} g_{n} B^{T} \hat{I}+S^{T} A \hat{I}+\hat{I}^{T} Q \hat{I}
$$

Where the terms in the Hamiltonian account for th electron Zeeman, the nuclear zeeman, the hyperfine, and nuclear quadrupole interactions, respectively. The reason we choose a spin doublet for the simulations is because cw-EPR data showed negligible interaction between the two unpaired electrons, supported by nutation data (see below). The optimised parameters are $A_{z}=4.322, A_{x}=0.351, A_{y}=0.0005 \mathrm{MHz}, K=0.484 \mathrm{MHz}$ and $\eta=0.750$. Using the same parameters one can successfully reproduce the main features of the 2-pulse ESEEM data. In this case, the additional peaks in the spectrum are likely to arise from band combinations, which are suppressed in a 3-pulse experiment by construction. The fitted parameters for the nuclear hyperfine are in line with literature values in related compounds. ${ }^{11-1213}$ 

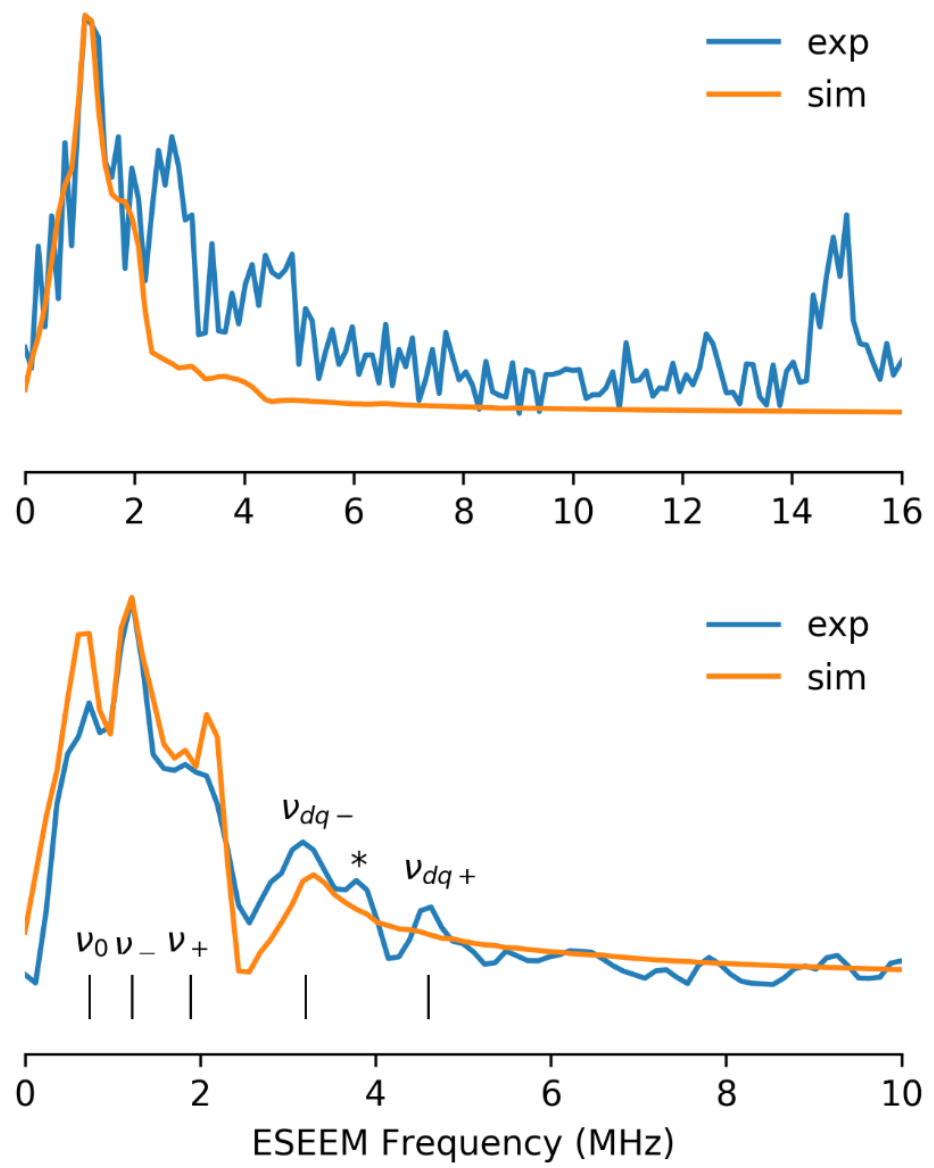

Figure S 17. X-band pulse EPR spectra of molecule 3 at $5 \mathrm{~K}$ at $352.9 \mathrm{mT}$ field position. Frequencydomain of 2-pulse (top) and 3-pulse(bottom) electron spin echo envelope modulation (ESEEM). * indicates the Larmor frequency of ${ }^{1} \mathrm{H}\left(\sim 15 \mathrm{MHz}\right.$, top) and ${ }^{13} \mathrm{C}$ ( $\sim 3.9 \mathrm{MHz}$, bottom), in the 2- and 3pulse experiment, respectively.

In an attempt to gain information on the spin state of the radicals and back up the results obtained from UV-Vis and NMR spectroscopy, transient nutations were collected on 3 using a two-pulse sequence with $t_{p}$ varying in 8 ns increments and 16 and 32 ns pulses. By Fourier transforming the time-domain data, one can extract the associated Rabi frequency ( $\omega_{\text {nut }}$, Figure S 18), which in the extreme weak limit for the microwave irradiation can be used to infere the spin multiplicity of the $\left|S, m_{S}\right\rangle \leftrightarrow\left|S, m_{S} \pm 1\right\rangle$ transitions ${ }^{14}$ from:

$$
\begin{array}{lr}
\omega_{\text {nut }}\left(m_{S}, m_{S+1}\right)=\frac{g_{1} \beta_{e} B_{1}}{\hbar} & (S=1 / 2) \\
\omega_{\text {nut }}\left(m_{S}, m_{S+1}\right)=\frac{g_{1} \beta_{e} B_{1}}{\hbar}\left[S(S+1)-m_{S}\left(m_{S}+1\right)\right]^{\frac{1}{2}} & (S \geq 1)
\end{array}
$$


with $g_{1}$ being the g-factor, $\beta_{e}$ the electronic bohr magneton, $\hbar$ the reduced Planck constant, $\quad B_{1}$ the microwave field, $S$ the total spin of the system and $m_{s}$ the spin sub-level involved in the transition. In our case, $\mathbf{B}_{1}$ is $5.575 \mathrm{mT}\left(\right.$ angle $=-\gamma_{e}\left|B_{1}\right| t_{p}$, having employed a 90 and 180 degrees pulse sequence and a $t_{p}=16 \mathrm{~ns}$ ). Table $\mathrm{S} 2$ compares the expected $\omega_{\text {nut }}$ values obtained from the $\left|S, m_{S}\right\rangle \leftrightarrow\left|S, m_{S} \pm 1\right\rangle$ expressions with those obtained from the Fourier transform of the nutation data, clearly indicating that the most reasonable agreement comes from a $S=1 / 2$ species. This justifies our choice of Hamiltonian to simulate the 3-pulse ESEEM data and further confirms that despite being an electronic triplet, the two unpaired electrons behave independently. From the EDFS spectrum (Figure S $10)$, the ratio between the g-values associated with the broad and sharp peaks is 1.029 ( $g=2.022$, $g=1.965$ for $\mathrm{H}_{\mathrm{dc}}=3454 \mathrm{G}, \mathrm{H}_{\mathrm{dc}}=3550 \mathrm{G}$, respectively). From a cw-EPR calibrated against strong pitch spectrum we know that the g-value associated with the only observed peak is 2.0069 - the g-value employed for calculating $\omega_{\text {nut }}$ are 2.065 and 2.0069.

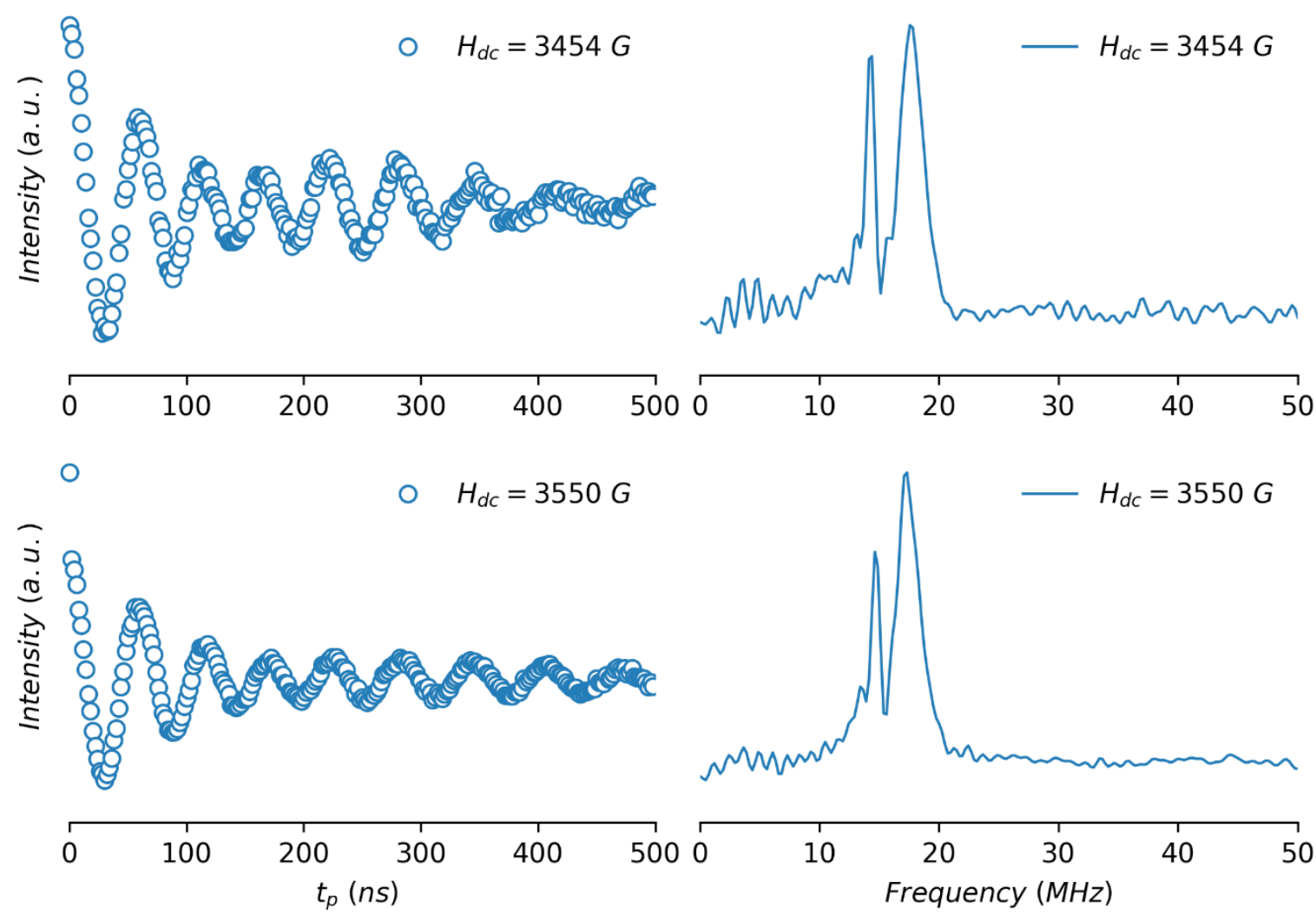

Figure S 18. Nutation experiments on 2 at $5 \mathrm{~K}$. Top and bottom plots correspond to $\mathrm{H}_{\mathrm{dc}}=3454$ and $\mathrm{H}_{\mathrm{dc}}$ $=3550 \mathrm{G}$ field positions, respectively. Right and left columns correspond to time- and frequencydomain. The peaks at $\sim 15$ and $\sim 17 \mathrm{MHz}$ are assigned to ${ }^{1} \mathrm{H}$ Larmor frequency and $\omega_{\text {nut }}$, respectively. 
Table S 2. Comparison of expected and calculated $\omega_{\text {nut }}$ in $\mathrm{MHz}$ for $\mathbf{2}$.

\begin{tabular}{ccc}
$\left|S, m_{S}\right\rangle \leftrightarrow\left|S, m_{S} \pm 1\right\rangle$ & $\mathrm{H}_{\mathrm{dc}}=3454 \mathrm{G}$ & $\mathrm{H}_{\mathrm{dc}}=3550 \mathrm{G}$ \\
\cline { 2 - 3 }$|1 / 2,1 / 2\rangle \leftrightarrow|1 / 2,-1 / 2\rangle$ & \multicolumn{3}{c}{$\omega_{\text {nut }}$} & $(\mathrm{MHz})$ \\
$|1,-1\rangle \leftrightarrow|1,0\rangle$ & 16.11 & 15.66 \\
& 22.79 & 22.15 \\
From FT of nutation & 17.68 & 17.20
\end{tabular}




\subsubsection{SQUID.}

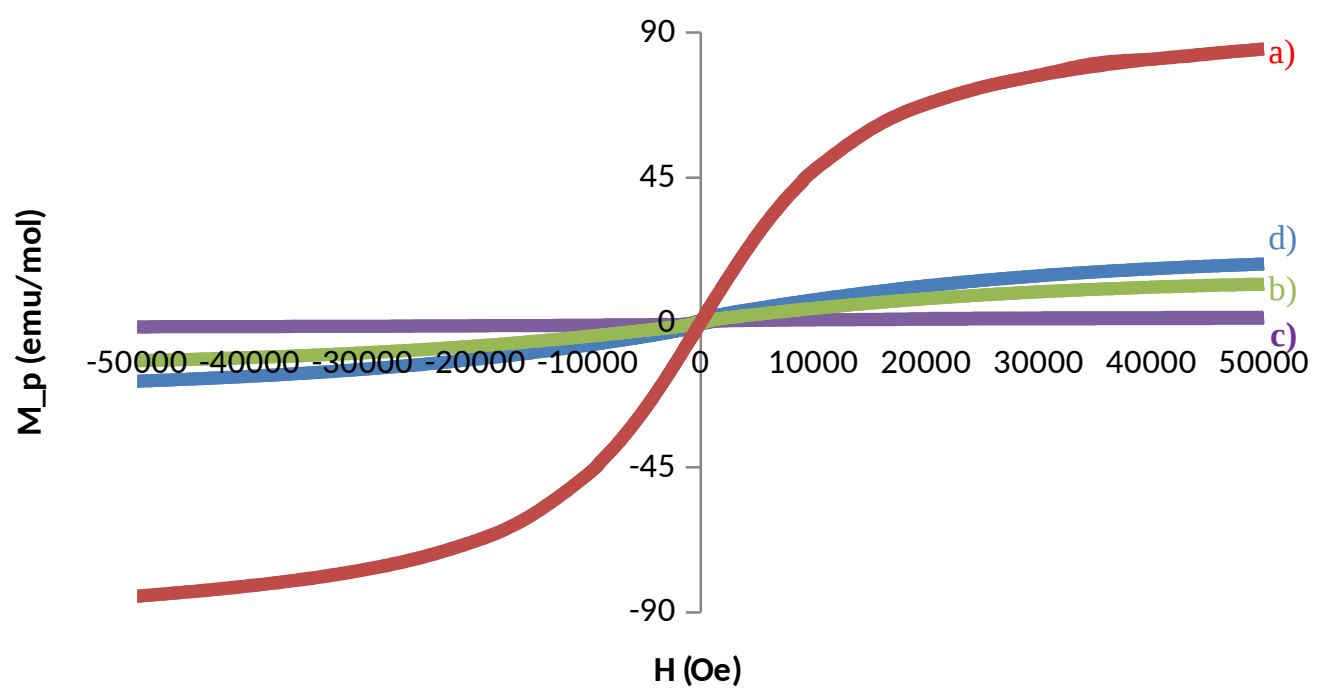

Figure S 19. Magnetization curves of $\mathbf{1}$ at $5 \mathrm{~K}$ of different powder samples: a) solid obtained by solvent elimination at reduced pressure b) solid obtained by recrystallization in acetone. c) solid obtained by sublimation. d) solid obtained by sublimation under infrared light. The solids in c) and d) are obtained from samples on a).

Magnetisation curves at $5 \mathrm{~K}$ of polycrystalline samples of molecule $\mathbf{5}$ are perfectly diamagnetic with a straight line with negative slope at all fields. 


\subsection{UV-Vis spectroscopy.}

Given that one can obtain equally good simulations of the EPR spectra assuming either a $S=1$ or $S=1 / 2$ model, extra information is required to distinguish between the two situations. For that, UV-Vis and NMR spectra were recorded in three scenarios: solution, after addition of the base DBU, and after neutralising the solution with acetic acid. If the origin of the paramagnetic species is a monoradical ( $S=1 / 2$ ), that process musth arise from a rupture of a molecular bond, which would result in chemical species that are very different before and after adding the base. UV-Vis and NMR are characterisation techniques perfectly suited to keep track of changes in solution between the different cases, directly addressing the feasibility of the models by chemical arguments.

UV-Vis spectra of molecules 1-4 are presented in Figure S 20-21. For molecules 1 and 2, the addition of base results in an intense band appearing at lower energies which completely disappears as the solution is neutralised with acetic acid, reverting to the original UV-Vis. How identical the UV-Vis spectrum of the acidified solution to the original one is, depends on how efficient the neutralisation has been. For instance, Figure S 20 shows an acidified solution with not enough equivalents of acid, and even if the broad absorption band at longer wavelengths has reverted, the lower wavelength region presents a mixture of both neutral and basic features. When more acid is added, one obtains an identical spectrum as compared to the original one. Note that this spectrum is not shown because we did not save it. For molecules $\mathbf{3}$ and $\mathbf{4}$, addition of the base and subsequent neutralisation does not introduce clear new absorption bands as in the case of $\mathbf{1}$ and 2, but there are still some noticeable changes. Molecule 3 shows partial deprotonation after addition of the base; the main peak $(450 \mathrm{~nm})$ recovers the maximum at the same wavelength as the neutral and loses the shoulder $(\sim 410$ $\mathrm{nm}$ ). Molecule 4 also shows partial deprotonation but the peak around $400 \mathrm{~nm}$ disappears when adding base and acid. No significative displacement of the main peak is observed. The bands associated with DBU are a broad absorption at $220 \mathrm{~nm}$ that ends below $280 \mathrm{~nm}$. Acetic acid does not show significant absorption bands above $280 \mathrm{~nm}$. However, their intensity is much lower than those related to the coloured compounds. Altogether, the UV-Vis spectra indicate that the species obtained after addition of the base keep the same properties as the molecule in solution and the process can be reverted by neutralization, indicating that the EPR signal cannot arise from the formation of molecular fragments. 


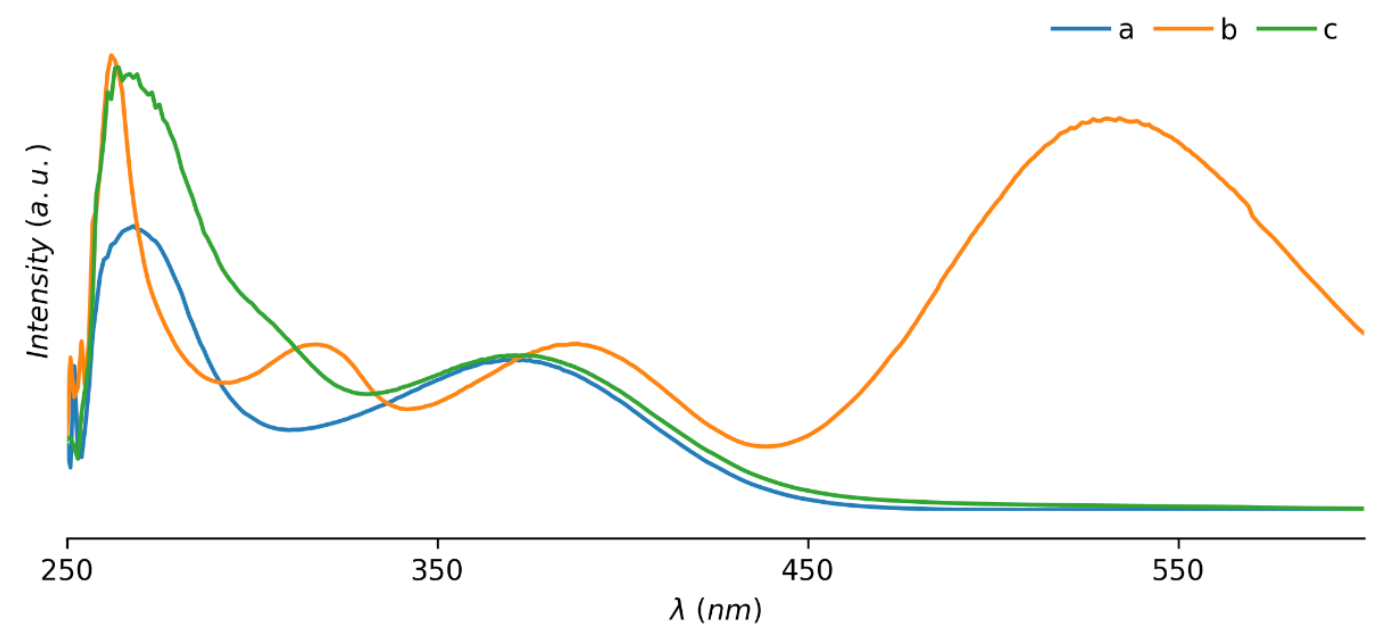

Figure S 20. UV-Vis spectra of molecule 1 in neutral (a), basic (b) and acidic (c) conditions.

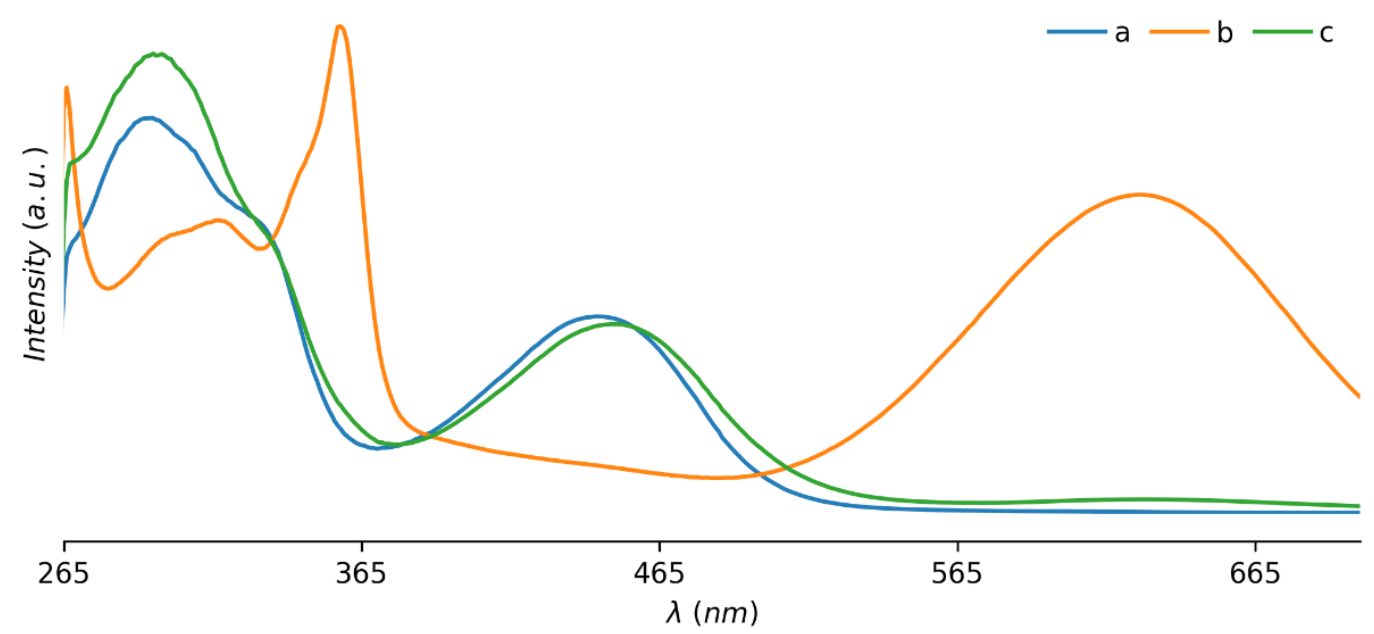

Figure S 21. UV-Vis spectra of molecule 2 in neutral (a), basic (b) and acidic (c) conditions.

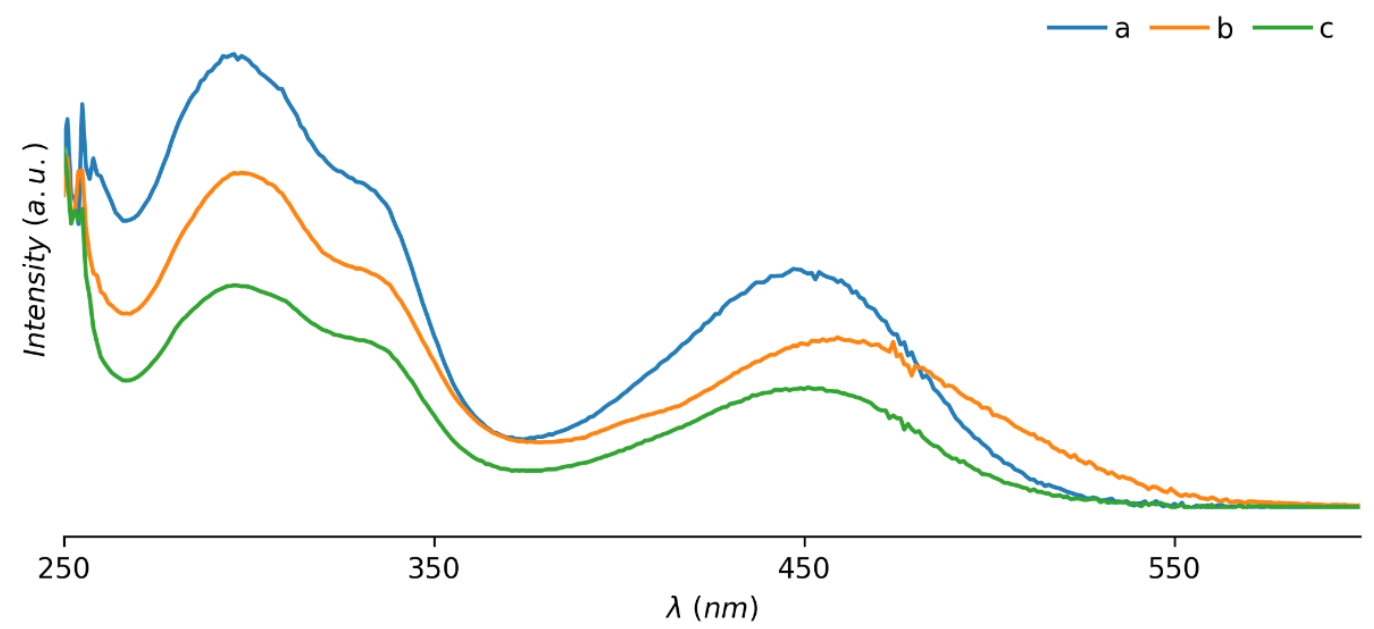

Figure S 22. UV-Vis spectra of molecules 3 in neutral (a), basic (b) and acidic (c) conditions. 


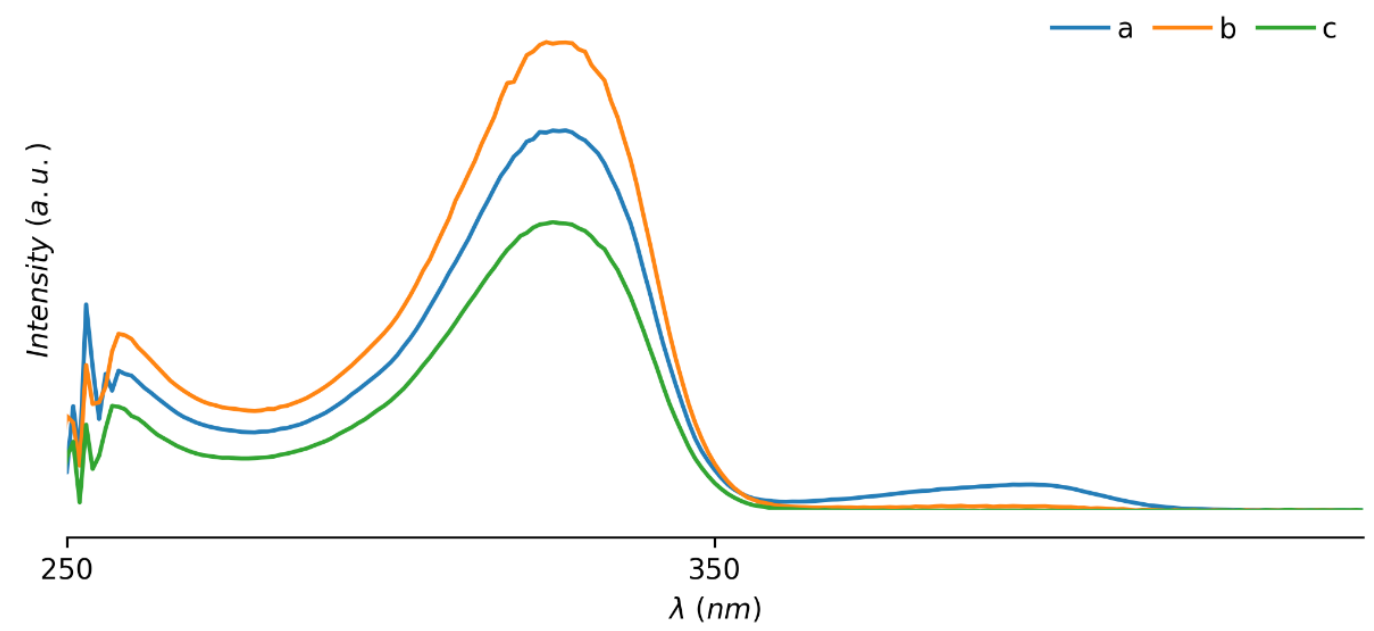

Figure S 23. UV-Vis spectra of molecule 4 in neutral (a), basic (b) and acidic (c) conditions.

Figure S 24 presents the change of colors for molecules 2 and 3. For all compounds, the colour of the solution changed when adding the base and was reverted when adding the acid. For molecules 1-3 the colour of the solution was orange, changing to dark purple (2) or dark red (3) with base; for Dasatinib, the solution was colourless, changing to a pale yellow after addition of the base.
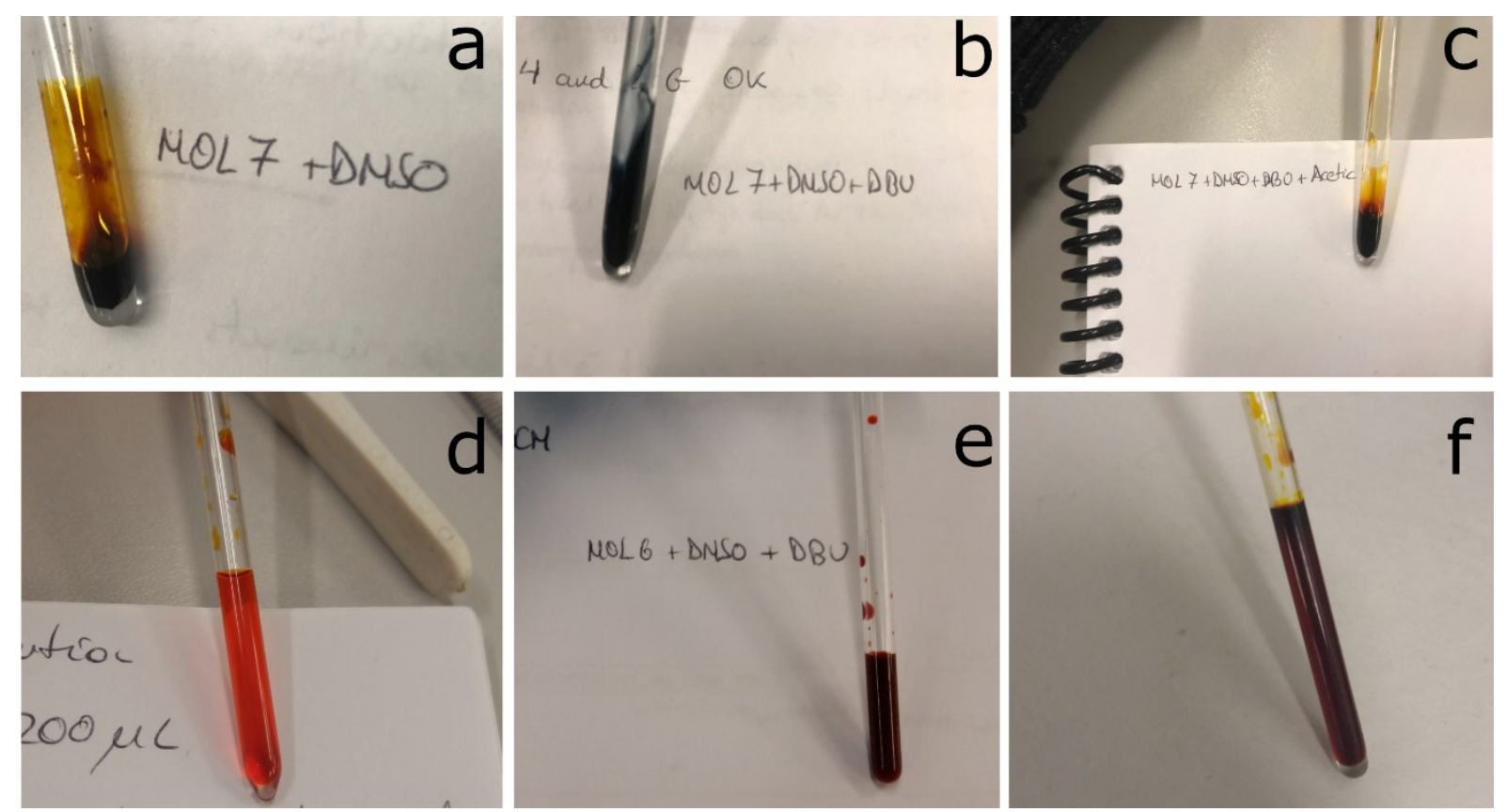

Figure S 24. Colour of the pure, basic and neutralised solution of molecules $\mathbf{2}$ and 3, a-c and d-f, respectively. 


\subsection{NMR.}

${ }^{1} \mathrm{H}$ NMR of 1, 2 and 4 were recorded for the pure solution (deuterated DMSO-d6), after base addition and after neutralisation with trifluoroacetic acid to simplify the NMR spectra. As compared to EPR and UV-Vis measurements, the base employed here is the conjugate of the solvent $\mathrm{DMSO}^{-}$and the acid is trifluoroacetic. The base and the acid were dissolved in deuterated DMSO-d6. We choose those to simplify the spectrum and avoid introducing extra protons that would overlap with the signals we want to track.

Figure S 25 presents the overlapped ${ }^{1} \mathrm{H}$ NMR spectra of $\mathbf{1}$ in the three situations. The initial spectrum corresponds to the expected structure of the protonated form. Addition of the strong base produces a different spectra that corresponds to the expected anion structure. Finally, addition of trifluoroacetic acid recovers the original peaks of the protonated structure. In all the cases, the broad peaks between 3 and 6 ppm correspond to coalescence peaks of the labile proton that can exchange with traces of water, the base and the acid. The peak at $2.5 \mathrm{ppm}$ corresponds to residual undeuterated DMSO. The fact that after neutralisation of the deprotonated form, one regains a spectrum with the same number of peaks that integrate to the same number of $\mathrm{H}$ atoms and shows the same number of splittings with the same $\mathrm{J}$ values proves that both compounds are the same chemical species. Additionally, no extra peaks are observed, precluding the possibility of decomposition of the deprotonated form since no new products are present. The differences in the chemical displacements and intensity are due to dilution after addition of the base and the acid (both solved in DMSO-d6) and to the significant increase of the ionic force in the final sample (Na trifluoroacetate). The differeces in chemical shifts of the corresponding peaks correlates with the change of polarity of the medium as expected (large for thiazole $\mathrm{H}$ atoms (two doublets around $7 \mathrm{ppm}$ ), small for the aromatic ring $\mathrm{H}$ atoms (singlet around $8 \mathrm{ppm}$ ), almost zero for the methyl $\mathrm{H}$ atoms (singlet near $2.2 \mathrm{ppm})$ ). 


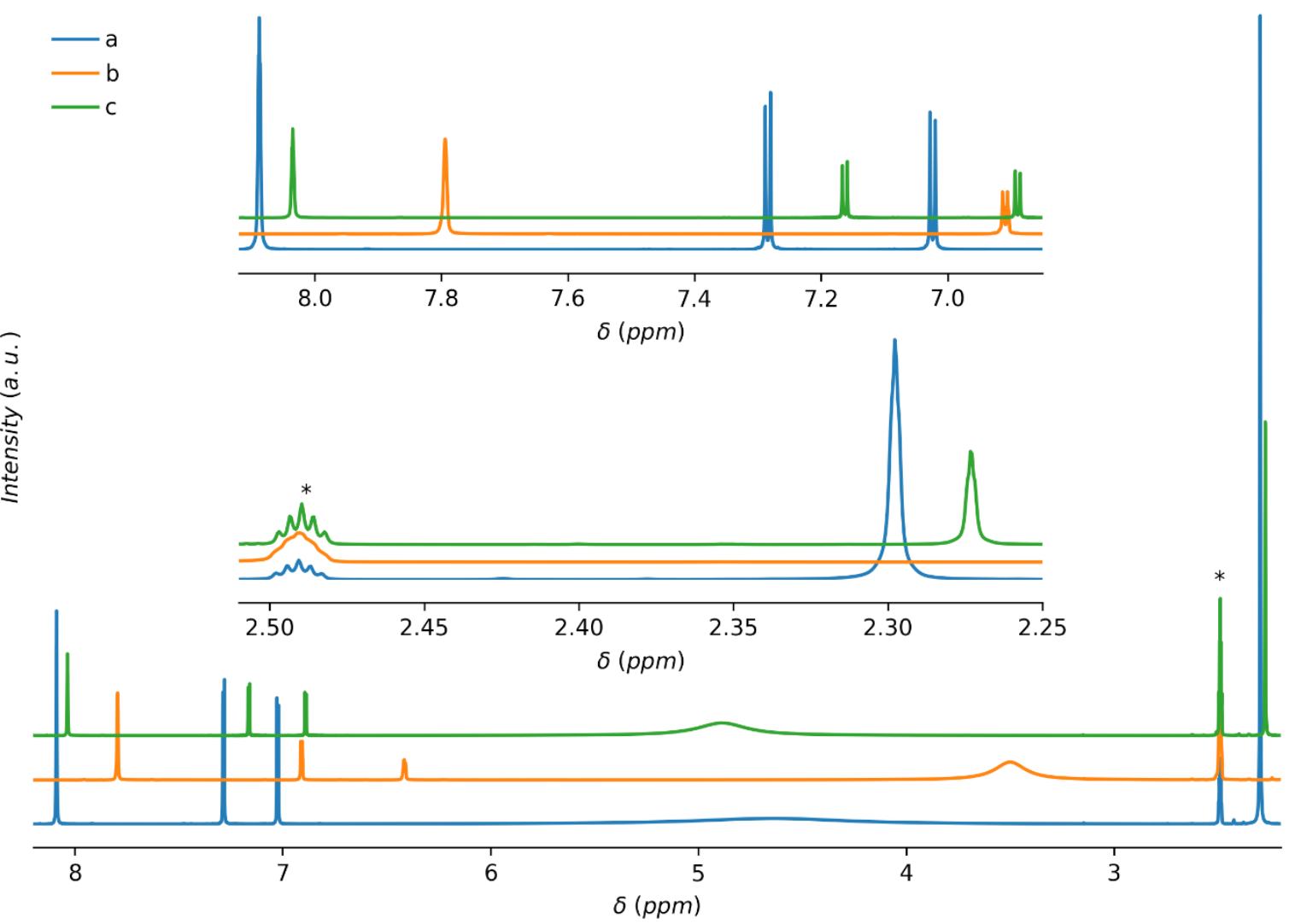

Figure S 25. ${ }^{1} \mathrm{H}$ NMR of $\mathbf{1}$ in DMSO-d6 (a), after addition of NaDMSO (b) and after neutralisation with trifluoroacetic acid (c). Subplots zoom in the different regions of the spectra. * indicates the protons of DMSO. a) (500 MHz, DMSO): $\delta(\mathrm{ppm})=8.10$ (s, $2 \mathrm{H}), 7.29(1 \mathrm{H}, \mathrm{d}, 4 \mathrm{~Hz}), 7.03(1 \mathrm{H}, \mathrm{d}, 4$ Hz), 2.31 (6 H, s). b) (500 MHz, DMSO): $\delta(\mathrm{ppm})=7.81$ (s, $2 \mathrm{H}), 6.92(1 \mathrm{H}, \mathrm{d}, 3,5 \mathrm{~Hz}), 6.42(1 \mathrm{H}, \mathrm{d}$, 2,5 Hz), 2.13 (6 H, s). c) (500 MHz, DMSO): $\delta$ (ppm) =8.05 (s, 2 H), 7.17 (1 H, d, 4 Hz), $6.90(1 \mathrm{H}$, d, $4 \mathrm{~Hz}), 2.28(6 \mathrm{H}, \mathrm{s})$.

${ }^{1} \mathrm{H}$ NMR of $\mathbf{2}$ and $\mathbf{3}$ were also recorded, but the larger number of $\mathrm{H}$ in the molecules makes the interpretation of the spectra more complicated. Still, we observe the same behaviour as discussed for the spectra of ${ }^{1} \mathrm{H}$ NMR spectrum of 1 . 


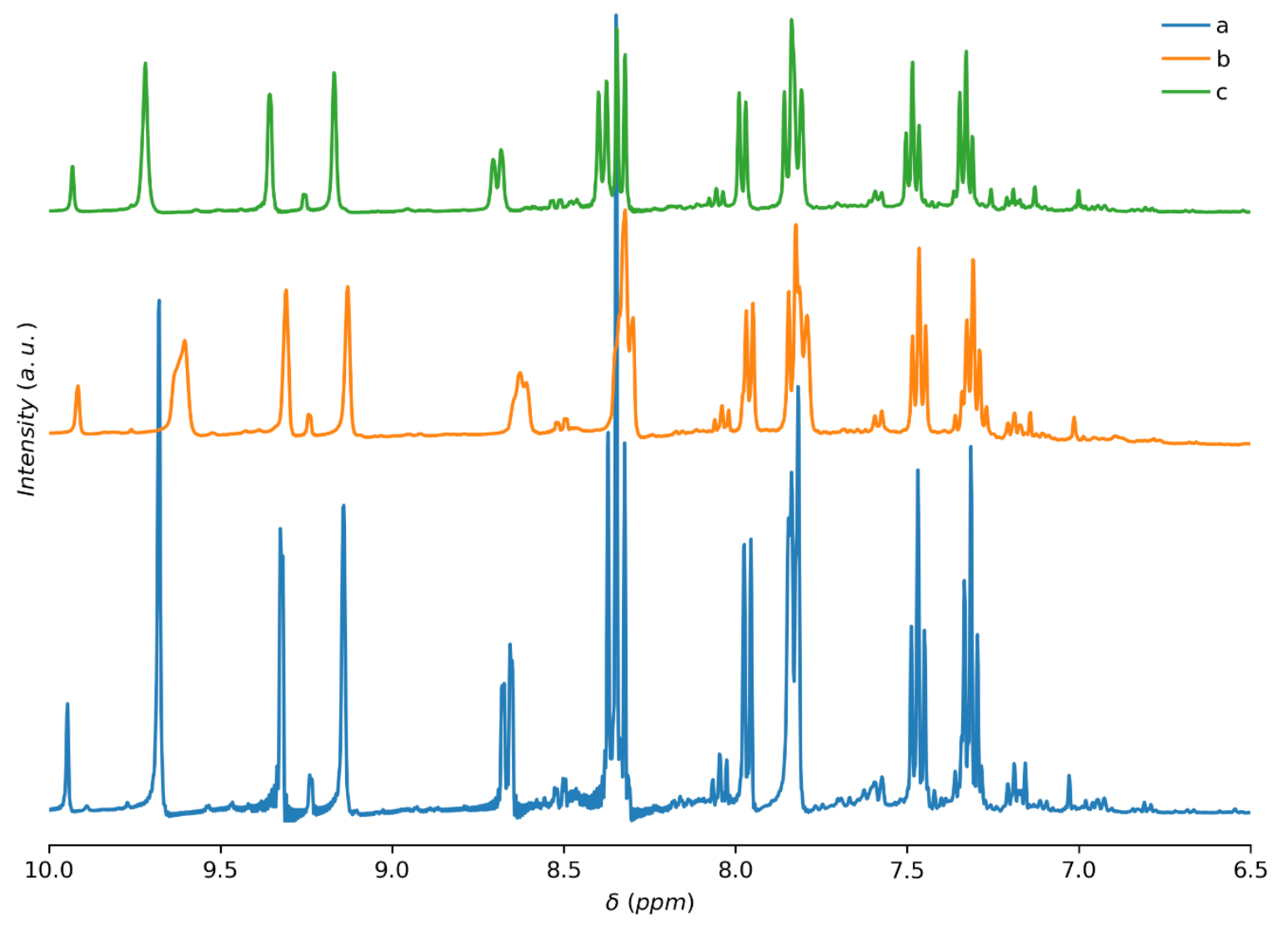

Figure S 26. ${ }^{1} \mathrm{H}$ NMR of $\mathbf{2}$ in DMSO-d6 (a), after addition of NaDMSO (b) and after neutralisation with trifluoroacetic acid (c). Partial deprotonation and reprotonation but no new peaks from sigma skeleton rearangement are observed. A new peak around $9.2 \mathrm{ppm}$ can be assigned to acridine protonation. 


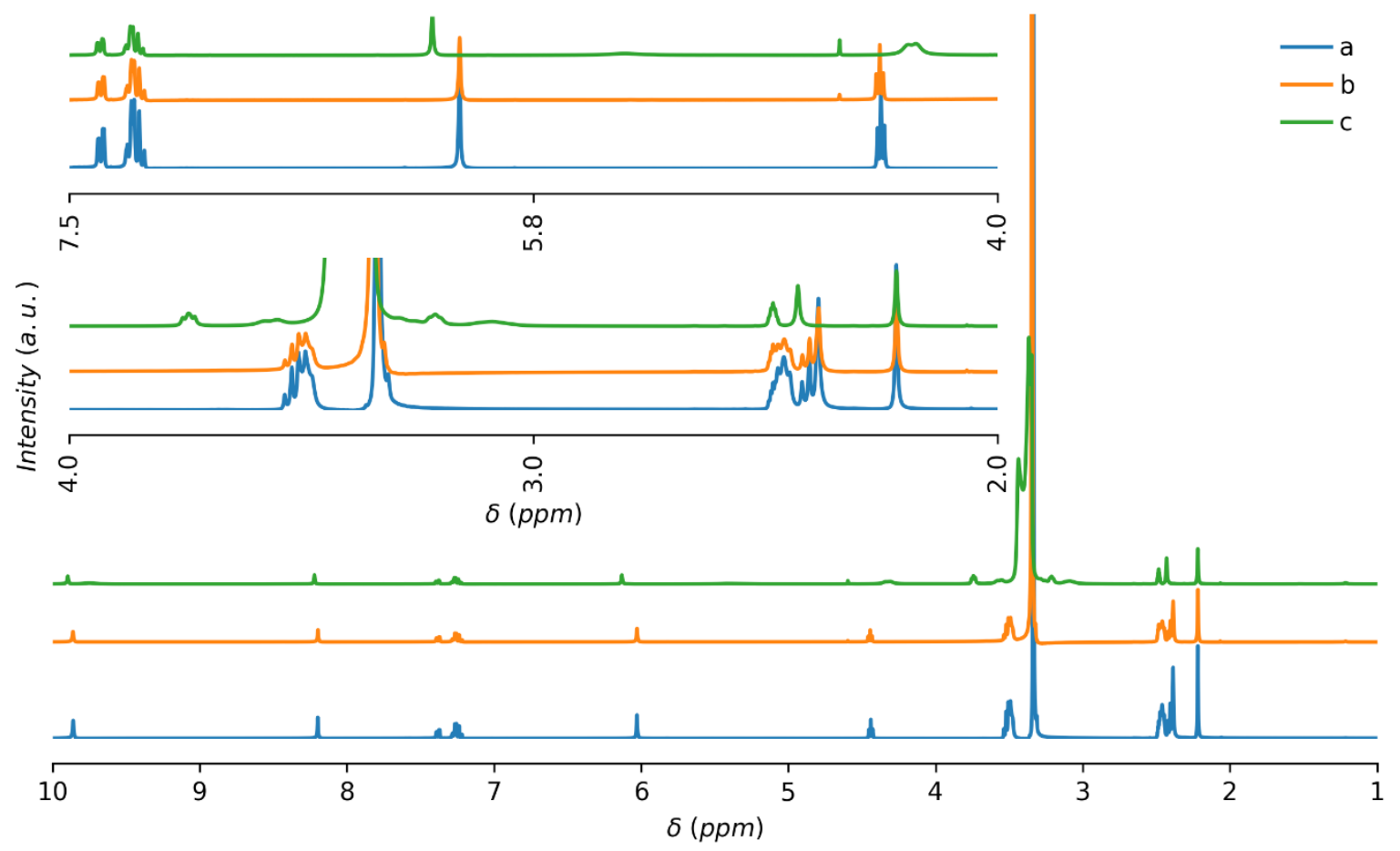

Figure S 27. ${ }^{1} \mathrm{H}$ NMR of 4 in DMSO-d6 (a), after addition of NaDMSO (b) and after neutralisation with trifluoroacetic acid (c). Partial deprotonation and reprotonation but no new peaks from sigma skeleton rearangement are observed. Small peaks around 10, 4.5 and $2.5 \mathrm{ppm}$ are likely due to other $\mathrm{N}$ positions being partially protonated.

Similarly, the ${ }^{13} \mathrm{C}$ NMR spectrum was also measured for $\mathbf{1}$ in the three conditions, observing an initial spectrum that corresponds to the expected structure of the protonated form. Addition of the strong base produces a different spectrum that corresponds to the expected anion structure. Finally, addition of trifluoroacetic acid recovers the original peaks of the protonated structure (+trifluoroacetic acid peaks). However, the spectra are not as clean as for ${ }^{1} \mathrm{H}$ NMR of $\mathbf{1}$ because of the large amount of different molecular carbon environments.

Taken altogether, it is clear that the addition of the base does not decompose the molecules as one obtains the same EPR, UV-Vis and NMR spectra when the medium is neutralised with an acid. This indicates that whatever radical species is formed, it cannot be a monoradical since this would lead to new subproducts after chemical reaction with surrounding molecules. We then conclude that the origin of the observed EPR signals must come from a molecular biradical (triplet electronic state). The mechanisms for its formation are explored in the next sections. 


\section{Topological valence bond interpretation of the biradical species.}

The amphoteric nature of these molecules is due to the imine-enamine tautomerism of the bridging nitrogen and the thiazole unit. This provides a mechanism for a small fraction of the molecules to generate an anion by proton transfer between two neutral molecules in the solid (proton transfer between two identical amphoteric molecules during the formation of the solid as $\begin{gathered}-i+A H^{i} \\ 2 A H \leftrightarrow A^{i}\end{gathered}$ ) or by the action of a base in an aprotic medium. The topology of the electron distribution of the anion allows for the formation of biradical species, which is stabilised with respect to the closed shell forms by the redistribution of the localised negative charge on the $\mathrm{N}$ bridging atom over the attractive $\mathrm{NO}_{2}$ group and a recovery of aromaticity. Furthermore, the structural flexibility of the bridge allows a perpendicular conformation of the two aromatic rings, resulting in a possible additional mechanism to stabilise the unpaired electrons due to orthogonality of the magnetic orbitals. In all cases, the biradical aromatic forms indicate a significant localisation and residence of the unpaired electrons on the $\mathrm{N}$ atoms of the NO2 group and amine bridge in compounds $\mathbf{1}$ and 2, whereas for compounds $\mathbf{3}$ and $\mathbf{4}$ a significant delocalization is expected for one of the unpaired electrons over several conjugated atoms. Here, the dominant resonant valence bond forms described in Scheme 1 of the main text for compound 1, are expanded here for molecules $\mathbf{2 - 4}$. For compound $\mathbf{2}$ the resulting valence bond forms are quite similar to those of compound $\mathbf{1}$ as shown in Scheme S29.

Figure S 28. Valence Bond forms in the ground state of the anion of compound 2. 
In the case of compound 3, at first sight the same reasoning cannot be applied since the methyl group substitutes the $\mathrm{H}$ atom and blocks the imine form of the imine-enamine tautomery observed in compound $\mathbf{2}$, leading to a molecule with no acidic protons. However, a detailed analysis of the valence bond forms of the protonated molecule shows that the methyl $\mathrm{H}$ atoms are indeed quite acidic due to inductive effects of the $\mathrm{NO}_{2}$ attracting group and the resonant effects of the thiazole and nitroacridine groups. A simple example that shows the relative importance of these effects is toluene and its para- cyano and nitro substituted analogs investigated by Bordwell et al. ${ }^{15}$ In this series, the reference structure toluene, has an estimated $\mathrm{pK}_{\mathrm{a}}$ of 42 (in DMSO, dissociation constant 31.8) whereas the cyano-substituted 4methylbenzonitrile has a $\mathrm{pK}_{\mathrm{a}}$ of 30 (inductive effect) and the nitro-substituted 1-methyl-4nitrobenzene, a $\mathrm{pK}_{\mathrm{a}}$ of 20.4 (inductive and resonance effects). Since $\mathrm{DBUH}^{+}$has an estimated $\mathrm{pK}_{\mathrm{a}}$ of ca. 12, the latter could be deprotonated by DBU in DMSO. In the case of compound 3, the larger the resonant parts of the molecule, the larger reduction of the $\mathrm{pK}_{\mathrm{a}}$ value due to the resonant effects as shown in the right VB form shown in Scheme S30. Both effects largely stabilise the anionic form of $\mathbf{3}$ compared to 1-methyl-4-nitrobenzene and, hence, can be easily deprotonated by DBU. In this case, a valence bond form increases the positive charge on the nearby $\mathrm{N}$ atom induced by recovering the aromaticity of the thiazole ring. This charge largely increases the acidity of the $\mathrm{H}$ atoms in the methyl group that can be extracted by a strong base such as DBU. The dominant valence bond forms in the ground state of $\mathbf{3}$ and the deprotonated form are depicted in Scheme S30. The mechanism to generate the anion species is different but the stabilisation of the biradical form is based on the same electronic processes as in the case of $\mathbf{1}$ and $\mathbf{2}$. 
Figure S 29. Valence Bond forms in the ground state of the anion of compound 3. In this case, the acidity of the $\mathrm{H}$ atoms of the methyl group (in green) is justified by the positive charge in the nearby $\mathrm{N}$ atom induced by recovering the aromaticity of the thiazole ring.

Here, the unpaired electron on the bridging $\mathrm{N}$ atom is expected to be more delocalised due to the participation of a secon biradical aromatic VB form that involves the conjugation with the deprotonated methyl group.

The dominant valence bond forms in the ground state of $\mathbf{4}$ and the deprotonated form are depicted in Scheme S31 The stabilisation of the biradical form in the deprotonated species is based on a similar electronic processes but the absence of a strong attractor group in the pyrimidinic group makes this molecule slightly different compared to the previous ones. However, a similar reasoning provides a justification of the acidity of the briging amine group by noticing that the amide group connected to the thiazole ring can also provide the inductive and resonant effects similar to the nitroacridine group although in a less pronounced than in compound 3. The resulting VB forms are depicted in Scheme S31 suggesting that a 
lesser acidity is expected for this compound that justifies the lower intensity observed in the EPR spectra.

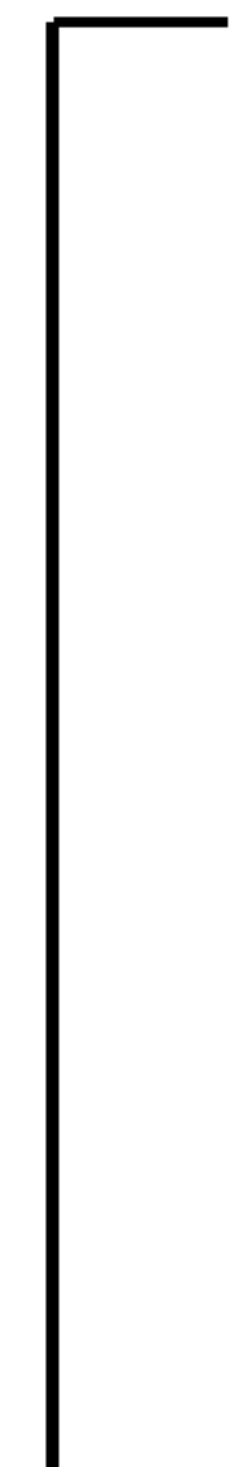

Figure S 30. Valence Bond forms in the ground state of the anion of compound 4.

It is worth to remark the following topological properties of the biradical VB forms compared to the initial aromatic form (with the negative charge on the $\mathrm{N}$ or $\mathrm{C}$ atom of the acidic group) and the charge-transfer quinoidal form in the anion: 1) the initially localised charge has been delocalised and the electronic repulsion is reduced; 2) negative charge is displaced to the most electroattractive 
groups; 3) aromaticity on the connecting ring is restored. In addition, the connection of these VB forms is performed by conjugation of most of the bonds involved in the charge transfer that also contribute to the delocalization of the unpaired electrons. These are, in our opinion, important electronic contributions that tend to stabilise the biradical form with respect to the closed shell forms that cannot be consistently described in a full valence CASSCF and correlated in a balenced form by, say, SD excitations due to the lack of symmetry introduced by the presence of heteroatoms (in contrast to Thiele's diradicaloid). 


\section{Electronic structure calculations and models.}

Multi-reference configuration interaction (MRCI) ${ }^{16}$ and complete active space self-consistent field (CASSCF) $)^{17,18}$ calculations were performed on molecule $\mathbf{1}$ as a representative system. All approaches employed to describe the energy difference between the singlet and triplet electronic states ( $\Delta E_{S-T}$ ) in molecule $\mathbf{1}$ are unable to predict a triplet ground state, failing to reproduce the experimental evidence. Depending on the method and basis sets used, we have obtained a dispersion of $\Delta E_{S_{0}-T}$ values, ranging from $\sim 70$ to $\sim 20 \mathrm{kcal} \cdot \mathrm{mol}^{-1}$, where the closed-shell singlet is always more stable. The reason for this erratic behaviour is that these molecules are dominated by a strong electronic correlation and a considerable electronnucleus coupling, effects that cannot be accurately described in these systems with the currently existing methods.

All electron calculations with large basis sets always overstabilise a closed shell solution in compound 1 and its deprotonated form. In common applications, it often suffices to treat the nuclei classically or to fix them to their equilibrium positions. Quantum nuclear effects such as tunneling, delocalization and zero-point energy, can be included later on by quantizing nuclear vibrations on the adiabatic ground state Born-Oppenheimer potential-energy surface. Ground-state electron-nuclear correlations beyond the Born-Oppenheimer approximation are however far beyond any existing electronic structure (either wave function or DFT based) method. When the nuclear variables and electron-nuclear coupling are treated exactly and fully quantum mechanically, the electrons feel, instead of an external potential, a "weighted" potential modified by the delocalization of the nuclear probability density as well as additional interactions induced by nonadiabatic electron-nuclear correlations. These effects are certainly not included in any existing electronic structure method, and therefore, while one could envision runing an extremely accurate electronic structure calculation, we believe that even that calculation would fail to describe molecule $\mathbf{1}$ due to the underlying electronnuclear correlation due to the construction scheme of (electron-electron and electron-nuclei) correlated wavefunctions. As a result of this correlation, there exists a crossing region where three kinds of interactions appear leading to a complex intersystem crossing including a conical intersection $\left(S_{0}-S_{1}\right)$, a seam $\left(S_{0}-T\right)$ and a quasidegeneracy between $S_{1}-S_{2}$ and $T$.

Detailed analysis of the wavefunction using different approaches (Complete Active Space Configuration Interaction CASCI with natural Broken Symmetry orbitals and a large number of correlated electrons/orbitals up to CAS $(12,12)$ ) show that small geometry changes have a large impact on the wavefunction. For instance, one finds that the relative weights of the associated valence bond coefficients change significantly, indicating a strong electron-nuclear coupling. To make things worse, the delocalised nature of the $\pi$ system and the presence of unpaired electrons is an extreme case in which strong electron correlation effects preclude the applicability of self consistent field approaches due to the size of the active space that one would have to use to introduce static correlation in a balanced manner for the triplet and singlet electronic states. This can be easily understood (Figure S32) by noticing that the open shell valence bond form in the $S_{z}=0$ determinant space is a single excitation of the closed 
shell determinant and, hence, both contribute to the wavefunctions of $S_{0}$ and $S_{1}$ of the anionic species.

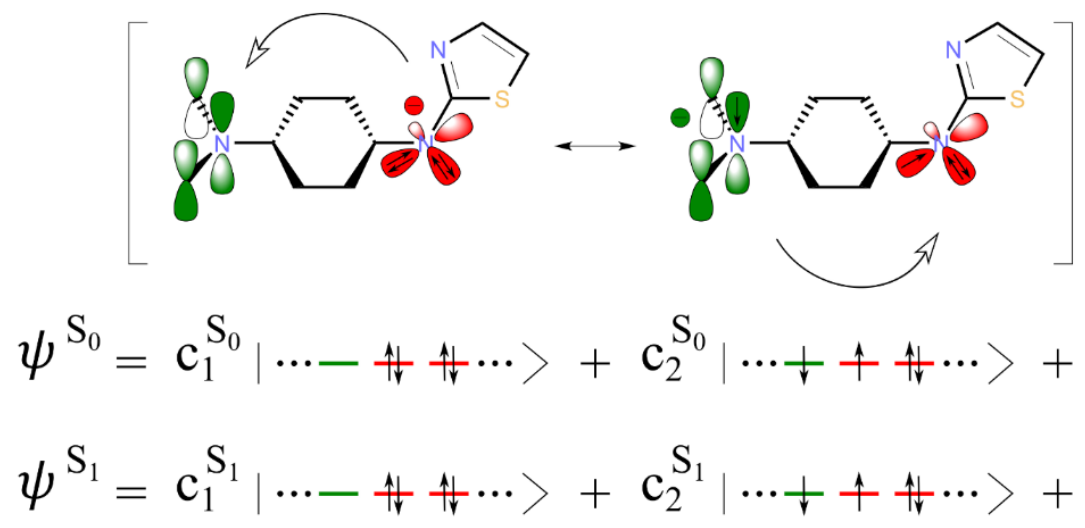

Figure S 32. Schematic representation of the strong electronic correlation effects in molecules 1-4.

In the case of $S_{0}, c_{1}^{S_{0}}$ contributes with the largest weight to the wavefunction, whereas in $S_{1}$ the main coefficient arises from $c_{1}^{S_{1}}$. Notice that these forms are related by single excitations and both contribute to the wavefuncions of $S_{0}$ and $S_{1}$ because they belong to the same $S_{z}$ and symmetry. This leads to one of the worst situations to be described by wavefunction methods since a) in HF the closed shell form is strongly overstabilised because by construction the method only describes the correlation of electrons with the same spin, b) in CASSCF solution the weight of single excitations of the reference are zero and the overstabilisation of closed shells is increased and c) for a given level of excitation over a set of orbitals (CAS+SD for instance), there are many more forms contributing to the $\mathrm{S}_{0}$ reference than to $S_{1}$, leading to an umbalanced description. Finally, all uncorrelated reference SCF functionals tend to underestimate aromaticity effects and the molecular orbitals of the phenyl ring have too high orbital energies and thus can mix preferably with closed shell forms and, again, favour $\mathrm{S}_{0}$.

The situation is even worse in DFT approaches since both HF and GGA favour closed shell solutions and, hence, we found overstabilised $S_{0}$ no matter the amount of Fock exchange that is included in the functional.

In conclusion, none of the available correlated wavefunctions are able to stabilise the openshell states by construction and, hence, are not useful to describe the experimentally observed situation. We have to rely on model systems and topological interpretations to understand the observed paramagnetic response.

Table S 3. $\left(E_{T}-E_{S}\right)$ energy differences (in $\mathrm{kcal} \cdot \mathrm{mol}^{-1}$ ) using a RHF/6-31G** reference geometry (Cs symmetry) with different BS (MIX: STO3G for C and H, 6-31G** for heteroatoms).

\begin{tabular}{|c|c|c|c|c|c|c|}
\hline $\boldsymbol{E}_{\boldsymbol{T}}-\boldsymbol{E}_{\boldsymbol{S}}$ & UHF/UHF & B3LYP & PBE0 & M06 & UwB97XD & LC-wPBE \\
\hline $\mathbf{6 - 3 1 g}$ ** & 36,8 & 58,3 & 58,5 & 59,7 & 61,1 & 61,2 \\
\hline MIX & 40,3 & 61,0 & 62,3 & 63,1 & 64,1 & 63,7 \\
\hline STO3G & 22,9 & 57,0 & 59,3 & 60,8 & 72,6 & 80,2 \\
\hline
\end{tabular}


Table S 4. $\left(E_{T}-E_{S}\right)$ energy differences (in $\mathrm{kcal} \cdot \mathrm{mol}^{-1}$ ) between optimised structures (using Cs symmetry) in each method and BS (MIX: STO3G for C and H, 6-31G** for heteroatoms).

\begin{tabular}{|c|c|c|c|c|c|c|}
\hline $\boldsymbol{E}_{\boldsymbol{T}}-\boldsymbol{E}_{\boldsymbol{S}}$ & UHF/RHF & B3LYP & PBE0 & M06 & uwB97XD & LC-wPBE \\
\hline $\mathbf{6 - 3 1 \mathbf { g } ^ { * * }}$ & 21,7 & 46,7 & 48,3 & 49,8 & 49,6 & 49,4 \\
\hline MIX & 22,8 & 46,1 & 48,7 & 50,1 & 48,8 & 48,0 \\
\hline STO3G & $-13,6$ & 23,2 & 23,8 & 36,1 & 22,6 & 20,4 \\
\hline
\end{tabular}

Table S 5. ( $\left(E_{T}-E_{S}\right)$ energy differences (in $\mathrm{kcal} \cdot \mathrm{mol}^{-1}$ ) using a RHF/6-31G** reference geometry (Cs symmetry) with different BS (MIX: STO3G for C and H, 6-31G** for heteroatoms).

\begin{tabular}{|c|c|c|c|}
\hline $\boldsymbol{E}_{\boldsymbol{T}} \boldsymbol{E}_{\boldsymbol{S}}$ & UHF/RHF & CAS1612 & DMRG \\
\hline 6-31g** & 36,8 & 76,1 & 40,0 \\
\hline MIX & 19,8 & 64,0 & -- \\
\hline STO3G & 28,3 & 35,0 & -- \\
\hline
\end{tabular}

HF/UHF apparently represents a nearby triplet using minimal basis sets, but none of the methods reproduce the observed biradical state when the BS is extended as is usually done to improve the results. In view of the limitations of the standard methods to represent a small $\boldsymbol{E}_{T}-\boldsymbol{E}_{S}$ energy difference in the anion as observed from magnetic experiments, a topological interpretation of the electronic structure based in an extention of the commonly accepted description of Thiele's and Tschitschibabin's diradicaloids has been developed to provide a consistent interpretation of the observed paramagnetic behaviour that we assign to biradical anions formed by deprotonation of the molecules 1-4. 
1. References. 
1S. L. Cockroft, J. Perkins, C. Zonta, H. Adams, S. E. Spey, C. M. R. Low, J. G. Vinter, K. R. Lawson, C. J. Urch and A. Hunter. Org. Biomol. Chem., 2007, 5, 1062-1080.

2A. Molinos-Gómez, X. Vidal, M. Maymó, D. Velasco, J. Martorell, F. López-Calahorra, Tetrahedron 2005, 61, $9075-9081$.

3S. Latorre, I. de P. R. Moreira, B. Villacampa, L. Julià, D. Velasco, J. M. Bofill and F. López-Calahorra, ChemPhysChem 2010, 11, 912-919.

4L. A. Pinck, G. E. Hilbert, J. Am. Chem. Soc., 1934, 56 (2), 490.

5A. Y. Liu, Q. Yang, D. Hao, W. Zhang, Aust. J. Chem., 2012, 65, 1390 - 1395.

6 N. F. Chilton, R. P. Anderson, L. D. Turner, A. Soncini and K. S. Murray J. Comput. Chem. 2013, 34, 11641175.

7 Wang, W.; Chen, C.; Shu, C.; Rajca, S.; Wang, X.; Rajca, A.; J. Am. Chem. Soc. 2018, 140, 25, 7820-7826 8 Schweiger, A.; Jeschke, G. Principles of Pulse Electron Paramagnetic Resonance. Oxford University Press, 2001.

9 Stoll, S.; Schweiger, A.; J. Magn. Reson. 2006, 178(1), 42-55.

10 Stoll, S.; Britt, R. D.; Phys. Chem. Chem. Phys. 2009, 11, 6614-6625.

11 Rabbani, S. R.; Edmonds, D. T.; Gosling, P.; Palmer, M. H. Journal of Magnetic Resonance 1987, 72, 2, 230-237.

12 Edmonds, D. T.; Speight, P. A. Physics Letters A 1971, 34, 6, 325-326

13 Taguchi, A. T.; O’Malley, P. J.; Wraight, C. A.; Dikanov, S. A.; J. Phys. Chem. B 2014, 118, 31, 92259237.

14 Equation 14.1.3 in ref 8..

15 F. G. Bordwell, D. Algrim, and N. R. Vanier. “Acidities of anilines and toluenes” J. Org. Chem. 42 (1977) 1817-1819

16 R. J. Gdanitz, R. Ahlrichs. Chem. Phys. Letters, 1988, 143:413-420.

17 B. O. Roos. Advances in Chemical Physics, 1987, 69, 399-445.

18 B. O. Roos. Int. J. Quantum Chem., 1980, 18-S14:175-189. 
